\title{
Qualitative Guest Sensing via Iron(II) Triazole Complexes
}

Santiago Rodríguez-Jiménez and Sally Brooker*

Department of Chemistry and the MacDiarmid Institute for Advanced Materials and Nanotechnology, University of Otago, PO Box 56, Dunedin 9054, New Zealand.

\section{SUPPORTING INFORMATION}

\section{Table of Contents}

S1. Single crystal structure analysis ......................................................................... 2

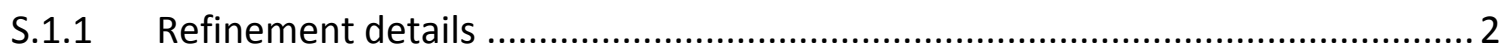

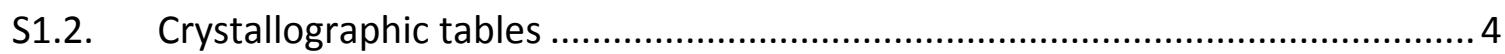

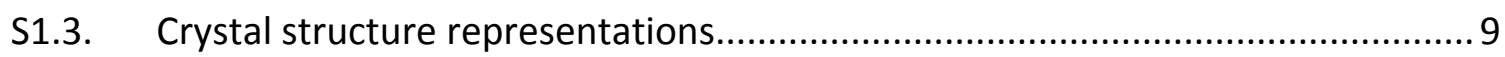

S1.4. Crystal structure representations of crystal packing ...................................... 12

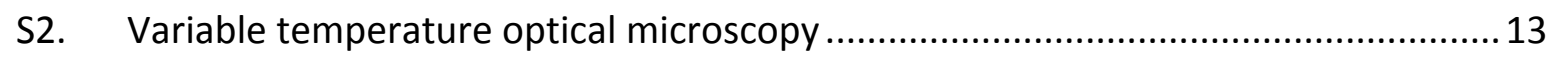

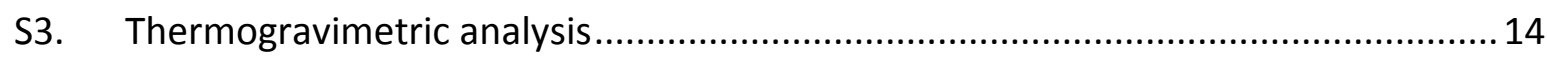

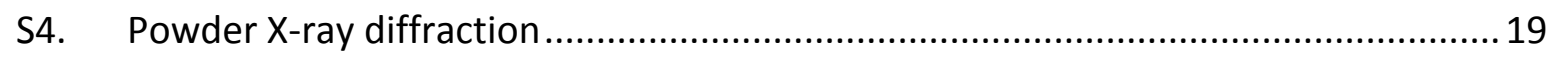

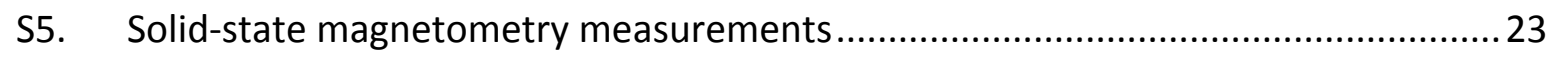




\section{S1. Single crystal structure analysis}

\section{S.1.1 Refinement details}

$\left[\mathrm{Fe}^{\prime \prime}\left(\mathrm{L}^{\text {pydzpy }}\right)_{\mathbf{2}}(\mathrm{NCS})_{2}\right](\mathbf{1})$. Half of this complex was found in the asymmetric unit; the other half was generated by a center of inversion found over the iron(II) atom. Residual e-density: +0.709 / - $0.779 e \cdot \AA^{-3}$. To certify the coordinated azine, and hence $C$ and $N$ atom positions were correctly assigned, comparisons of the values of the anisotropic displacement parameters $\left(\mathrm{U}_{\text {eq }}\right)$ obtained from refining the structure with different combinations of possible $\mathrm{N}$ and $\mathrm{C}$ atom assignments between the coordinated and uncoordinated rings were carried out. In addition, the number of Q-peaks high in the difference map found adjacent to the pyridazine (3 Q-peaks) and pyridine (4 Q-peaks) rings, and the location of them, was consistent with the hydrogen atom positions for the $C$ assignments, and with the conclusions reached from the $\mathrm{U}_{\mathrm{eq}}$ comparisons. This enabled clear identification of both (i) the pyridazine (uncoordinated) and pyridine (coordinated) rings, and (i) the orientations of these rings with respect to the triazole ring.

[Fe"( (pydzpy $_{2}$ (NCSe) $)_{2}$ (2). Half of this complex was found in the asymmetric unit, the other half was generated by a center of inversion located at the iron(II) atom. Residual e-density: +0.939 / $-0.606 \mathrm{e} \cdot \AA^{-3}$. The above-described methodology was used to certify the coordinated azine ring confirming that pyridine, and not pyridazine, is coordinated to the iron(II) centre.

[Fe"( $\left.\left(\mathrm{L}^{\text {pydzpy }}\right)_{2}(\mathrm{NCS})_{2}\right] \cdot 4 \mathrm{CH}_{3} \mathrm{CN}\left(1 \cdot 4 \mathrm{CH}_{3} \mathrm{CN}\right)$ at $100 \mathrm{~K}$. Half of this complex was found in the asymmetric unit, the other half was generated by a center of inversion located at the iron(II) atom. Two ordered lattice $\mathrm{CH}_{3} \mathrm{CN}$ solvent molecules were found within the asymmetric unit. All non-hydrogen atoms were refined anisotropically. Residual e-density: $+0.576 /-0.462 \mathrm{e} \cdot \AA^{-3}$. The above-described methodology was used to certify the coordinated azine ring confirming that pyridine, and not pyridazine, is coordinated to the iron(II) centre.

[Fe"( $\left.\left(\mathrm{L}^{\text {pydzpy }}\right)_{2}(\mathrm{NCS})_{2}\right] \cdot 4 \mathrm{CH}_{3} \mathrm{CN}\left(1 \cdot 4 \mathrm{CH}_{3} \mathrm{CN}\right)$ at $225 \mathrm{~K}$. Half of this complex was found in the asymmetric unit, the other half was generated by a center of inversion located at the iron(II) atom. Two lattice acetonitrile solvent molecules were found within the asymmetric unit, the first $\mathrm{CH}_{3} \mathrm{CN}$ is ordered, while the second $\mathrm{CH}_{3} \mathrm{CN}$ is disordered over two positions (35:65 - N9 C22 C23/N10 C24 C25). All non-hydrogen atoms were refined anisotropically, but disordered 
$\mathrm{CH}_{3} \mathrm{CN}$ of solvation. Residual e-density: $+0.320 /-0.527 \mathrm{e} \cdot \AA^{-3}$. The above-described methodology was used to certify the coordinated azine ring confirming that pyridine, and not pyridazine, is coordinated to the iron(II) centre.

$\left[\mathrm{Fe}^{\prime \prime}\left(\mathrm{L}^{\text {pydzpy }}\right)_{2}(\mathrm{NCSe})_{2}\right] \cdot 4 \mathrm{CH}_{3} \mathrm{CN}\left(2 \cdot 4 \mathrm{CH}_{3} \mathrm{CN}\right)$ at 100 and $270 \mathrm{~K}$. Half of this complex was found in the asymmetric unit, the other half was generated by a center of inversion located at the iron(II) atom. Two ordered lattice $\mathrm{CH}_{3} \mathrm{CN}$ molecules were found within the asymmetric unit. All non-hydrogen atoms were refined anisotropically. Residual e-density@100 K: +0.397/0.395 e. $\AA^{-3}$; @270K: +0.435/ -0.470 e. $\AA^{-3}$. The above-described methodology was used to certify the coordinated azine ring confirming that pyridine, and not pyridazine, is coordinated to the iron(II) centre. 


\section{S1.2. Crystallographic tables}

Table S1. Selected crystallographic data of complexes $\left[\mathrm{Fe}^{\prime \prime}\left(\mathrm{L}^{\text {pydzpy }}\right)_{2}(\mathrm{NCS})_{2}\right] \quad$ (1) and $\left[\mathrm{Fe}^{\prime \prime}\left(\mathrm{L}^{\text {pydzpy }}\right)_{2}(\mathrm{NCSe})_{2}\right](\mathbf{2})$ at $100 \mathrm{~K},\left[\mathrm{Fe}^{\prime \prime}\left(\mathrm{L}^{\text {pydzpy }}\right)_{2}(\mathrm{NCS})_{2}\right] \cdot 4 \mathrm{CH}_{3} \mathrm{CN}\left(\mathbf{1} \cdot 4 \mathrm{CH}_{3} \mathrm{CN}\right)$ at 100 and $225 \mathrm{~K}$, and $\left[\mathrm{Fe}^{\prime \prime}\left(\mathrm{L}^{\text {pydzpy }}\right)_{2}(\mathrm{NCS})_{2}\right] \cdot 4 \mathrm{CH}_{3} \mathrm{CN}\left(\mathbf{2} \cdot 4 \mathrm{CH}_{3} \mathrm{CN}\right)$ at 100 and $270 \mathrm{~K}$.

\begin{tabular}{|c|c|c|c|c|c|c|}
\hline & 1 & 2 & $1.4 \mathrm{CH}_{3} \mathrm{CN}$ & $1.4 \mathrm{CH}_{3} \mathrm{CN}$ & $2 \cdot 4 \mathrm{CH}_{3} \mathrm{CN}$ & $2 \cdot 4 \mathrm{CH}_{3} \mathrm{CN}$ \\
\hline Temperature (K) & $100(2)$ & $100(2)$ & $100(2)$ & $225(2)$ & $100(2)$ & $270(2)$ \\
\hline Empirical formula & $\mathrm{C}_{38} \mathrm{H}_{28} \mathrm{~N}_{14} \mathrm{~S}_{2} \mathrm{Fe}$ & $\mathrm{C}_{38} \mathrm{H}_{28} \mathrm{~N}_{14} \mathrm{Se}_{2} \mathrm{Fe}$ & $\mathrm{C}_{46} \mathrm{H}_{40} \mathrm{~N}_{18} \mathrm{~S}_{2} \mathrm{Fe}$ & $\mathrm{C}_{46} \mathrm{H}_{40} \mathrm{~N}_{18} \mathrm{~S}_{2} \mathrm{Fe}$ & $\mathrm{C}_{46} \mathrm{H}_{40} \mathrm{~N}_{18} \mathrm{Se}_{2} \mathrm{Fe}$ & $\mathrm{C}_{46} \mathrm{H}_{40} \mathrm{~N}_{18} \mathrm{Se}_{2} \mathrm{Fe}$ \\
\hline$M$ & 800.71 & 894.51 & 964.93 & 964.93 & 1058.73 & 1058.73 \\
\hline Crystal system & Triclinic & Triclinic & Monoclinic & Monoclinic & Triclinic & Triclinic \\
\hline Space group & $P-1$ & $P-1$ & $P 2_{1} / n$ & $P 2_{1} / n$ & $P-1$ & $P-1$ \\
\hline$a[\AA ̊]$ & $8.8022(3)$ & $8.8922(3)$ & $7.69148(15)$ & $7.8170(2)$ & $7.5538(4)$ & $7.6962(4)$ \\
\hline$b[\AA ̊]$ & $9.0691(3)$ & $9.1156(3)$ & $12.9758(3)$ & $13.1050(3)$ & $11.8013(8)$ & $12.0272(7)$ \\
\hline$c[\AA ̊]$ & $11.5779(5)$ & $11.5757(4)$ & $23.4345(4)$ & $23.6246(5)$ & $13.5691(9)$ & $13.7862(5)$ \\
\hline$\alpha\left[^{\circ}\right]$ & $76.415(3)$ & $75.956(3)$ & 90 & 90 & $82.785(5)$ & $83.221(4)$ \\
\hline$B\left[^{\circ}\right]$ & $76.705(3)$ & $76.873(3)$ & $91.8905(15)$ & $91.073(2)$ & $87.176(5)$ & $86.089(4)$ \\
\hline$\gamma\left[^{\circ}\right]$ & $85.680(3)$ & $84.849(3)$ & 90 & 90 & $73.640(5)$ & $71.600(5)$ \\
\hline$V\left[\AA^{3}\right]$ & $874.09(6)$ & $885.88(5)$ & $2337.57(7)$ & $2419.71(10)$ & $1151.33(13)$ & $1201.75(11)$ \\
\hline Z & 1 & 1 & 2 & 2 & 1 & 1 \\
\hline$\rho_{\text {calcd. }}\left[\mathrm{Mg} \cdot \mathrm{m}^{3}\right]$ & 1.521 & 1.677 & 1.371 & 1.324 & 1.527 & 1.463 \\
\hline Wavelength $[\AA ̊]$ & 0.71073 & 0.71073 & 1.54184 & 1.54184 & 0.71073 & 0.71073 \\
\hline$\mu\left[\mathrm{mm}^{-1}\right]$ & 0.601 & 2.533 & 3.869 & 3.737 & 1.964 & 1.882 \\
\hline$F(000)$ & 412 & 448 & 1000 & 1000 & 536 & 536 \\
\hline Crystal size $\left[\mathrm{mm}^{3}\right]$ & $\begin{array}{l}0.189 \times 0.065 \times \\
0.046\end{array}$ & $\begin{array}{l}0.220 \times 0.069 \times \\
0.046\end{array}$ & $\begin{array}{l}0.313 \times 0.070 x \\
0.046\end{array}$ & $\begin{array}{l}0.313 \times 0.070 x \\
0.046\end{array}$ & $\begin{array}{l}0.133 \times 0.031 \times \\
0.026\end{array}$ & $\begin{array}{l}0.232 \times 0.049 \times \\
0.020\end{array}$ \\
\hline $\begin{array}{l}\theta \text { range: data } \\
\text { collection [ㅇ] }\end{array}$ & 3.216 to 29.651 & 3.176 to 26.022 & 3.774 to 73.576 & 3.743 to 73.604 & 3.027 to 26.021 & 3.049 to 26.019 \\
\hline Reflections collected & 15864 & 14274 & 12882 & 12511 & 18834 & 19317 \\
\hline $\begin{array}{l}\text { Independent } \\
\text { reflections }\end{array}$ & 4361 & 3473 & 4612 & 4744 & 4540 & 4718 \\
\hline$R$ (int) & 0.0274 & 0.0236 & 0.0467 & 0.0537 & 0.0673 & 0.0396 \\
\hline $\begin{array}{l}\text { Max./min. } \\
\text { transmission }\end{array}$ & 1 and 0.77191 & 0.947 and 0.646 & 1 and 0.65572 & 1 and 0.56303 & $\begin{array}{l}1 \text { and } \\
0.82187\end{array}$ & 0.963 and 0.669 \\
\hline $\begin{array}{l}\text { Data/restraints / } \\
\text { parameters }\end{array}$ & 4361 / 0 / 251 & 3473 / 0 / 251 & 4612 / 0 / 307 & 4744 / 0 / 303 & 4540 / 0 / 306 & 4718 / 0 / 306 \\
\hline $\begin{array}{l}\text { Max/min e- density } \\
{\left[e \AA^{-1}\right]}\end{array}$ & $\begin{array}{l}0.709 \text { and - } \\
0.779\end{array}$ & 0.934 and -0.614 & $\begin{array}{l}0.576 \text { and - } \\
0.462\end{array}$ & $\begin{array}{l}0.320 \text { and - } \\
0.527\end{array}$ & 0.397 and -0.395 & 0.435 and -0.470 \\
\hline Goof $\left(F^{2}\right)$ & 1.076 & 1.059 & 1.160 & 1.167 & 1.029 & 1.080 \\
\hline $\mathrm{R}_{1} / w \mathrm{R}_{2}[\mathrm{I}>2 \sigma(\mathrm{I})]$ & $\begin{array}{l}R_{1}=0.0482, \\
w R_{2}=0.1390\end{array}$ & $\begin{array}{l}R_{1}=0.0413, \\
W R_{2}=0.1209\end{array}$ & $\begin{array}{l}\mathrm{R}_{1}=0.0496, \\
w R_{2}=.1387\end{array}$ & $\begin{array}{l}\mathrm{R}_{1}=0.0570, \\
w R_{2}=.1738\end{array}$ & $\begin{array}{l}R_{1}=0.0356, \\
w R_{2}=0.0731\end{array}$ & $\begin{array}{l}R_{1}=0.0408, \\
w R_{2}=0.0858\end{array}$ \\
\hline$w \mathrm{R}_{2}$ [all data] & 0.1426 & 0.1218 & 0.1426 & 0.1837 & 0.0766 & 0.0909 \\
\hline
\end{tabular}


Table S2. Selected bond distances and angles in structures of $\left[\mathrm{Fe}^{\prime \prime}\left(\mathrm{L}^{\text {pydzpy }}\right)_{2}(\mathrm{NCS})_{2}\right](\mathbf{1}),\left[\mathrm{Fe}^{\prime \prime}\left(\mathrm{L}^{\text {pydzpy }}\right)_{2}(\mathrm{NCSe})_{2}\right](\mathbf{2}),\left[\mathrm{Fe}^{\prime \prime}\left(\mathrm{L}^{\mathrm{pydzpy}}\right)_{2}(\mathrm{NCS})_{2}\right] \cdot 4 \mathrm{CH}{ }_{3} \mathrm{CN}\left(\mathbf{1} \cdot 4 \mathrm{CH} \mathrm{C}_{3} \mathrm{CN}\right)$ at 100 and $225 \mathrm{~K}$, and $\left[\mathrm{Fe}^{\prime \prime}\left(\mathrm{L}^{\text {pydzpy }}\right)_{2}(\mathrm{NCSe})_{2}\right] \cdot 4 \mathrm{CH}_{3} \mathrm{CN}\left(\mathbf{2} \cdot 4 \mathrm{CH}_{3} \mathrm{CN}\right)$ at 100 and $270 \mathrm{~K}$.

\begin{tabular}{|c|c|c|c|c|c|c|}
\hline & 1 & 2 & $1 \cdot 4 \mathrm{CH}_{3} \mathrm{CN}$ & & $2 \cdot 4 \mathrm{CH}_{3} \mathrm{CN}$ & \\
\hline Temperature / K & $100(2)$ & $100(2)$ & $100(2)$ & $225(2)$ & $100(2)$ & $270(2)$ \\
\hline Fe1-N1 / ^̊ & $2.1974(19)$ & 2.191(3) & $2.027(2)$ & $2.202(3)$ & $2.025(2)$ & $2.211(2)$ \\
\hline Fe1-N2 / Å & $2.1864(18)$ & $2.180(3)$ & $1.953(3)$ & $2.121(3)$ & $1.979(2)$ & $2.150(2)$ \\
\hline Fe1-N7 / ̊̊ & $2.120(3)$ & $2.132(4)$ & $1.968(2)$ & $2.107(4)$ & $1.944(2)$ & $2.108(3)$ \\
\hline Fe1-N1a / Å & $2.1974(19)$ & 2.191(3) & $2.027(2)$ & $2.202(3)$ & $2.025(2)$ & $2.211(2)$ \\
\hline Fe1-N2a / Å & $2.1864(18)$ & $2.180(3)$ & $1.953(3)$ & $2.121(3)$ & $1.979(2)$ & $2.150(2)$ \\
\hline Fe1-N7a / & $2.120(3)$ & $2.132(4)$ & $1.968(2)$ & $2.107(4)$ & $1.944(2)$ & $2.108(3)$ \\
\hline N1-Fe1-N2 / & $74.68(7)$ & $74.98(11)$ & $90.17(11)$ & $74.87(11)$ & 79.65(9) & 74.59(9) \\
\hline N1-Fe1-N7 / ${ }^{\circ}$ & $85.45(8)$ & $94.28(12)$ & $79.62(10)$ & $89.16(13)$ & 91.36(9) & $91.30(10)$ \\
\hline N1-Fe1-N1a / & 180.00 & 180.00 & 180.00 & 180.00 & 180.00 & 180.00 \\
\hline N1-Fe1-N2a / & $105.32(7)$ & $105.02(11)$ & $89.83(11)$ & $105.13(11)$ & $100.35(9)$ & 105.41(9) \\
\hline N1-Fe1-N7a / ${ }^{\circ}$ & $94.55(8)$ & $85.72(12)$ & $100.38(10)$ & $90.84(13)$ & $88.64(9)$ & $88.70(10)$ \\
\hline N2-Fe1-N7 / & $86.21(9)$ & $93.18(13)$ & $88.57(11)$ & $91.29(13)$ & $92.27(9)$ & $92.56(9)$ \\
\hline N2-Fe1-N1a / & $105.32(7)$ & $105.02(11)$ & $89.83(11)$ & $105.13(11)$ & $100.35(9)$ & 105.41(9) \\
\hline N2-Fe1-N2a / ${ }^{\circ}$ & 180.00 & 180.00 & 180.00 & 180.00 & 180.00 & 180.00 \\
\hline N2-Fe1-N7a / & 93.79(9) & $86.82(13)$ & $91.43(11)$ & $88.71(13)$ & $87.73(9)$ & $87.44(9)$ \\
\hline N7-Fe1-N1a / & $94.55(8)$ & $85.72(12)$ & $100.38(10)$ & $90.84(13)$ & $88.64(9)$ & $88.70(10)$ \\
\hline N7-Fe1-N2a / & 93.79(9) & $86.82(13)$ & $91.43(11)$ & $88.71(13)$ & 87.73(9) & $87.44(9)$ \\
\hline N7-Fe1-N7a / & 180.00 & 180.00 & 180.00 & 180.00 & 180.00 & 180.00 \\
\hline N1a-Fe1-N2a / ${ }^{\circ}$ & $74.68(7)$ & $74.98(11)$ & $90.17(11)$ & $74.87(11)$ & 79.65(9) & 74.59(9) \\
\hline N1a-Fe1-N7a / ${ }^{\circ}$ & $85.45(8)$ & $94.28(12)$ & $79.62(10)$ & $89.16(13)$ & $91.36(9)$ & $91.30(10)$ \\
\hline N2a-Fe1-N7a / ${ }^{\circ}$ & $86.21(9)$ & $93.18(13)$ & $88.57(11)$ & $91.29(13)$ & $92.27(9)$ & $92.56(9)$ \\
\hline
\end{tabular}




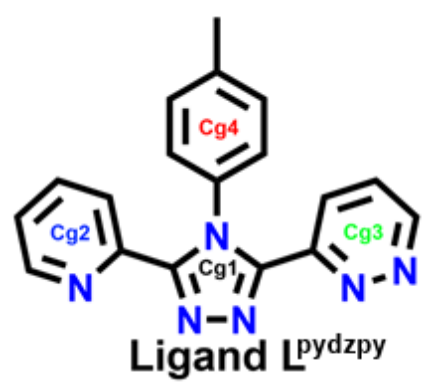

Figure S1. Schematic representation of ligand $L^{\text {pydzpy }}$ showing each ring and its corresponding centroid. Centroid color code: triazole ring is Cg1 (black), pyridine ring is Cg2 (blue), pyridazine ring is Cg3 (green), and tolyl ring is Cg4 (red).

Table S3. Selected intermolecular contacts in the structure of $\left[\mathrm{Fe}^{\prime \prime}\left(\mathrm{L}^{\text {pydzpy }}\right)_{2}(\mathrm{NCS})_{2}\right](\mathbf{1})$ at $100 \mathrm{~K}$.

\begin{tabular}{|c|c|c|c|c|}
\hline \multicolumn{5}{|c|}{ Compound $\left[\mathrm{Fe}^{\prime \prime}\left(\mathrm{L}^{\text {pydzpy }}\right)_{2}(\mathrm{NCS})_{2}\right](\mathbf{1})$} \\
\hline D-H...A & $\mathrm{D}-\mathrm{H} / \AA \AA$ & H...A / $\AA$ & D...A / $\AA$ & $\mathrm{D}-\mathrm{H} \ldots \mathrm{A} /{ }^{\circ}$ \\
\hline $\mathrm{C} 13-\mathrm{H} 13 \ldots \mathrm{N} 3$ & 0.95 & 2.53 & $3.442(3)$ & 161 \\
\hline C18-H18A...N5 & 0.98 & 2.62 & $3.570(3)$ & 164 \\
\hline Centroid...Centroid & & $\mathrm{Cg} \ldots \mathrm{Cg} / \AA$ & & angle $\mathrm{Cg} . . . \mathrm{Cg} /{ }^{\circ}$ \\
\hline Cg1...Cg1 & & $3.8272(12)$ & & $0(12)$ \\
\hline $\operatorname{Cg} 2 \ldots \operatorname{cg} 3$ & & $4.3070(14)$ & & $34.63(12)$ \\
\hline Cg3....cg3 & & $3.8125(14)$ & & $0.02(12)$ \\
\hline X-H...Centroid & & $\mathrm{H} . . . \mathrm{Cg} / \AA$ & $\mathrm{X} \ldots \mathrm{Cg} / \AA$ & $\mathrm{X}-\mathrm{H} \ldots \mathrm{Cg} /{ }^{\circ}$ \\
\hline $\mathrm{C} 4-\mathrm{H} 4 \ldots \mathrm{Cg} 4$ & & 2.82 & $3.665(3)$ & 149 \\
\hline NCS...SCN & & S...S / $\AA$ & & \\
\hline $\mathrm{S} 1 \ldots \mathrm{S} 1 \mathrm{~A}$ & & $3.918(3)$ & & \\
\hline
\end{tabular}

Table S4. $\quad$ Selected intermolecular contacts in the structure of $\left[\mathrm{Fe}^{\prime \prime}\left(\mathrm{L}^{\text {pydzpy }}\right)_{2}(\mathrm{NCSe})_{2}\right](\mathbf{2})$ at $100 \mathrm{~K}$.

\begin{tabular}{|c|c|c|c|c|}
\hline \multicolumn{5}{|c|}{ Compound $\left[\mathrm{Fe}^{\prime \prime}\left(\mathrm{L}^{\text {pydzpy }}\right)_{2}(\mathrm{NCSe})_{2}\right](\mathbf{5})$} \\
\hline D-H...A & $\mathrm{D}-\mathrm{H} / \AA ̊$ & H....A / ̊ & D...A / $\AA$ & $\mathrm{D}-\mathrm{H} \ldots \mathrm{A} /{ }^{\circ}$ \\
\hline $\mathrm{C} 13-\mathrm{H} 13 \ldots \mathrm{N3}$ & 0.95 & 2.54 & $3.460(5)$ & 164 \\
\hline C18-H18A...N5 & 0.98 & 2.64 & $3.600(5)$ & 166 \\
\hline Centroid...Centroid & & $\mathrm{Cg} \ldots \mathrm{Cg} / \AA$ & & angle $\mathrm{Cg} \ldots \mathrm{Cg} /{ }^{\circ}$ \\
\hline $\operatorname{Cg} 1 \ldots \operatorname{Cg} 1$ & & $3.926(2)$ & & $0(19)$ \\
\hline $\mathrm{Cg} 2 \ldots \mathrm{Cg} 3$ & & $4.377(2)$ & & $36.28(18)$ \\
\hline $\mathrm{Cg} 3 \ldots \mathrm{Cg} 3$ & & $3.876(2)$ & & $0.02(18)$ \\
\hline $\mathrm{X}$-H...Centroid & & H....Cg / $\AA$ & X....Cg / & $\mathrm{X}-\mathrm{H} \ldots \mathrm{Cg} /{ }^{\circ}$ \\
\hline C4-H4...Cg4 & & 2.82 & $3.664(4)$ & 149 \\
\hline NCSe...NCSe & & $\mathrm{Se} . . . \mathrm{Se} / \AA ̊$ & & \\
\hline Se1...Se1A & & $3.609(4)$ & & \\
\hline
\end{tabular}


Table S5. Selected intermolecular contacts in the structure of $\left[\mathrm{Fe}^{\prime \prime}\left(\mathrm{L}^{\text {pydzpy }}\right)_{2}(\mathrm{NCS})_{2}\right] \cdot 4 \mathrm{CH}_{3} \mathrm{CN}$ $\left(1 \cdot 4 \mathrm{CH}_{3} \mathrm{CN}\right)$ at $100 \mathrm{~K}$.

\begin{tabular}{|c|c|c|c|c|}
\hline \multicolumn{5}{|c|}{ Compound $\left[\mathrm{Fe}^{\prime \prime}\left(\mathrm{L}^{\text {pydzpy }}\right)_{2}(\mathrm{NCS})_{2}\right] \cdot 4 \mathrm{CH}_{3} \mathrm{CN}\left(1 \cdot 4 \mathrm{CH}_{3} \mathrm{CN}\right)$} \\
\hline \multicolumn{5}{|c|}{$100 \mathrm{~K}$} \\
\hline D-H...A & $\mathrm{D}-\mathrm{H} / \AA$ & H...A / $\AA$ & D...A / A & $D-H \ldots A /{ }^{\circ}$ \\
\hline $\mathrm{C} 4-\mathrm{H} 4 \ldots \mathrm{N} 3$ & 0.95 & 2.30 & $3.124(5)$ & 144 \\
\hline $\mathrm{C} 12-\mathrm{H} 12 \ldots \mathrm{S} 1$ & 0.95 & 2.85 & $3.581(4)$ & 135 \\
\hline C15-H15...N6 & 0.95 & 2.39 & $3.267(5)$ & 153 \\
\hline Centroid...Centroid & & $\mathrm{Cg} \ldots \mathrm{Cg} / \AA ̊$ & & angle $\mathrm{Cg} . . . \mathrm{Cg} /{ }^{\circ}$ \\
\hline Cg1...Cg2 & & $4.5190(17)$ & & $2.55(16)$ \\
\hline $\mathrm{Cg} 2 \ldots \mathrm{Cg} 3$ & & $3.9349(18)$ & & $8.98(16)$ \\
\hline Y-X...Cg & & X...Cg / Å & Y...Cg / Å & $\mathrm{Y}-\mathrm{X} \ldots \mathrm{Cg} /{ }^{\circ}$ \\
\hline C22-N9...Cg1 & & $3.394(3)$ & $3.466(4)$ & $84.1(2)$ \\
\hline
\end{tabular}

Table S6. Selected intra-, and intermolecular contacts in the structure of $\left[\mathrm{Fe}^{\prime \prime}\left(\mathrm{L}^{\text {pydzpy }}\right)_{2}(\mathrm{NCS})_{2}\right] \cdot 4 \mathrm{CH}_{3} \mathrm{CN}\left(\mathbf{1} \cdot 4 \mathrm{CH}_{3} \mathrm{CN}\right)$ at $225 \mathrm{~K}$.

\begin{tabular}{|c|c|c|c|c|}
\hline \multicolumn{5}{|c|}{ Compound $\left[\mathrm{Fe}^{\prime \prime}\left(\mathrm{L}^{\text {pydzpy }}\right)_{2}(\mathrm{NCS})_{2}\right] \cdot 4 \mathrm{CH}_{3} \mathrm{CN}\left(\mathbf{1} \cdot 4 \mathrm{CH}_{3} \mathrm{CN}\right)$} \\
\hline \multicolumn{5}{|c|}{$225 \mathrm{~K}$} \\
\hline D-H...A & $\mathrm{D}-\mathrm{H} / \AA$ & H...A / $\AA$ & D...A / $\AA$ & $\mathrm{D}-\mathrm{H} \ldots \mathrm{A} /{ }^{\circ}$ \\
\hline $\mathrm{C} 3-\mathrm{H} 3 . . . \mathrm{N} 10$ & 0.94 & 2.62 & $3.300(9)$ & 130 \\
\hline C9-H9...N3 (intra) & 0.94 & 2.48 & $2.790(6)$ & 100 \\
\hline $\mathrm{C} 17-\mathrm{H} 17 \ldots \mathrm{N} 10$ & 0.94 & 2.49 & $3.372(8)$ & 156 \\
\hline C17-H17...N9 & 0.94 & 2.33 & $3.258(15)$ & 171 \\
\hline $\mathrm{C} 25-\mathrm{H} 25 \mathrm{~B} \ldots \mathrm{N} 6$ & 0.97 & 2.47 & $3.375(12)$ & 154 \\
\hline Centroid...Centroid & & $\mathrm{Cg} \ldots \mathrm{Cg} / \AA$ & & angle $\mathrm{Cg} \ldots \mathrm{Cg} /{ }^{\circ}$ \\
\hline $\operatorname{Cg} 1 \ldots \operatorname{cg} 2$ & & $4.610(2)$ & & $4.70(18)$ \\
\hline $\operatorname{cg} 2 \ldots \operatorname{cg} 3$ & & $3.944(2)$ & & $9.48(19)$ \\
\hline $\mathrm{X}-\mathrm{H} . .$. Centroid & & $\mathrm{H} . . . \mathrm{Cg} / \AA ̊ ̊$ & $\mathrm{X} \ldots \mathrm{Cg} / \AA$ & $\mathrm{X}-\mathrm{H} . . . \mathrm{Cg} /{ }^{\circ}$ \\
\hline $\mathrm{C} 4-\mathrm{H} 4 \ldots \mathrm{Cg} 4$ & & 2.88 & $3.729(4)$ & 151 \\
\hline $\mathrm{C} 23-\mathrm{H} 23 \mathrm{C} . . . \mathrm{Cg} 4$ & & 2.87 & $3.726(17)$ & 147 \\
\hline Y-X...Cg & & $\mathrm{X} \ldots \mathrm{Cg} / \AA$ & Y...Cg / $\AA$ & $\mathrm{Y}-\mathrm{X} \ldots \mathrm{Cg} /{ }^{\circ}$ \\
\hline C20-N8...Cg1 & & $3.416(4)$ & $3.482(5)$ & $83.8(3)$ \\
\hline
\end{tabular}


Table S7. Selected intra-, and intermolecular contacts in the structure of $\left[\mathrm{Fe}^{\prime \prime}\left(\mathrm{L}^{\text {pydzpy }}\right)_{2}(\mathrm{NCSe})_{2}\right] \cdot 4 \mathrm{CH}_{3} \mathrm{CN}\left(\mathbf{2} \cdot 4 \mathrm{CH}_{3} \mathrm{CN}\right)$ at $100 \mathrm{~K}$.

\begin{tabular}{|c|c|c|c|c|}
\hline \multicolumn{5}{|c|}{ Compound $\left[\mathrm{Fe}^{\prime \prime}\left(\mathrm{L}^{\text {pydzpy }}\right)_{2}(\mathrm{NCSe})_{2}\right] \cdot 4 \mathrm{CH}_{3} \mathrm{CN}\left(2 \cdot 4 \mathrm{CH}_{3} \mathrm{CN}\right)$} \\
\hline \multicolumn{5}{|c|}{$100 \mathrm{~K}$} \\
\hline D-H...A & $\mathrm{D}-\mathrm{H} / \AA$ & H...A / $\AA$ & D...A / $\AA$ & $\mathrm{D}-\mathrm{H} \ldots \mathrm{A} /{ }^{\circ}$ \\
\hline C9-H9...Se1 & 0.95 & 2.89 & $3.662(3)$ & 139 \\
\hline $\mathrm{C} 1-\mathrm{H} 1 \ldots \mathrm{N} 3$ (intra) & 0.95 & 2.34 & 3.149(3) & 143 \\
\hline C11-H11...N8 & 0.95 & 2.70 & $3.460(3)$ & 137 \\
\hline Centroid...Centroid & & $\mathrm{Cg} \ldots \mathrm{Cg} / \AA$ & & angle $\mathrm{Cg} \ldots \mathrm{Cg} /{ }^{\circ}$ \\
\hline Cg1 ...cg2 & & $4.4629(15)$ & & $5.27(13)$ \\
\hline $\operatorname{Cg} 1 \ldots \operatorname{Cg} 3$ & & $4.8364(16)$ & & $2.09(13)$ \\
\hline $\operatorname{Cg} 2 \ldots \operatorname{cg} 3$ & & $3.7688(14)$ & & $6.64(12)$ \\
\hline $\mathrm{X}-\mathrm{H} . .$. Centroid & & $\mathrm{H} \ldots \mathrm{Cg} / \AA$ & X...Cg / $\AA$ & $\mathrm{X}-\mathrm{H} \ldots \mathrm{..Cg} /{ }^{\circ}$ \\
\hline $\mathrm{C} 4-\mathrm{H} 4 \ldots \mathrm{Cg} 4$ & & 2.84 & $3.682(3)$ & 148 \\
\hline Y-X...Cg & & $\mathrm{X} \ldots \mathrm{..Cg} / \AA ̊ \AA$ & Y...Cg / $\AA$ & $\mathrm{Y}-\mathrm{X} \ldots \mathrm{Cg} /{ }^{\circ}$ \\
\hline C22-N9...Cg4 & & $3.626(4)$ & $4.093(3)$ & 121.4(3) \\
\hline
\end{tabular}

Table S8. Selected intermolecular contacts in the structure of [Fe" $\left.\left(\mathrm{L}^{\text {pydzpy }}\right)_{2}(\mathrm{NCSe})_{2}\right] \cdot 4 \mathrm{CH}{ }_{3} \mathrm{CN}$ $\left(2 \cdot 4 \mathrm{CH}_{3} \mathrm{CN}\right)$ at $270 \mathrm{~K}$.

\begin{tabular}{|c|c|c|c|c|}
\hline \multicolumn{5}{|c|}{ Compound $\left[\mathrm{Fe}^{\prime \prime}\left(\mathrm{L}^{\text {pydzpy }}\right)_{2}(\mathrm{NCSe})_{2}\right] \cdot 4 \mathrm{CH}_{3} \mathrm{CN}\left(2 \cdot 4 \mathrm{CH}_{3} \mathrm{CN}\right)$} \\
\hline \multicolumn{5}{|c|}{$270 \mathrm{~K}$} \\
\hline D-H...A & $\mathrm{D}-\mathrm{H} / \AA$ & H...A / $\AA$ & D...A / $\AA$ & $\mathrm{D}-\mathrm{H} \ldots \mathrm{A} /{ }^{\circ}$ \\
\hline C1-H1...N3 (intra) & 0.93 & 2.49 & $2.794(4)$ & 100 \\
\hline C11-H11...N8 & 0.93 & 2.70 & $3.459(5)$ & 139 \\
\hline C13-H13...N9 & 0.93 & 2.67 & $3.336(5)$ & 129 \\
\hline Centroid...Centroid & & $\mathrm{Cg} \ldots \mathrm{Cg} / \AA$ & & angle $\mathrm{Cg} \ldots \mathrm{Cg} /{ }^{\circ}$ \\
\hline Cg1...Cg2 & & $4.6516(18)$ & & $9.85(15)$ \\
\hline Cg1...Cg3 & & $4.8956(19)$ & & $3.21(16)$ \\
\hline $\operatorname{Cg} 2 \ldots \operatorname{Cg} 3$ & & $3.7702(19)$ & & $6.75(16)$ \\
\hline X-H...Centroid & & $\mathrm{H} . . . \mathrm{Cg} / \AA$ & $\mathrm{X} \ldots \mathrm{Cg} / \AA$ & $\mathrm{X}-\mathrm{H} \ldots \mathrm{Cg} /{ }^{\circ}$ \\
\hline $\mathrm{C} 4-\mathrm{H} 4 \ldots \mathrm{Cg} 4$ & & 2.84 & $3.6555(3)$ & 146 \\
\hline $\mathrm{Y}-\mathrm{X} \ldots \mathrm{Cg}$ & & $\mathrm{X} . . . \mathrm{Cg} / \AA$ & Y...Cg / A & $\mathrm{Y}-\mathrm{X} \ldots \mathrm{Cg} /{ }^{\circ}$ \\
\hline C20-N8...Cg4 & & $3.965(3)$ & $4.314(4)$ & $88.4(2)$ \\
\hline
\end{tabular}




\section{S1.3. Crystal structure representations}

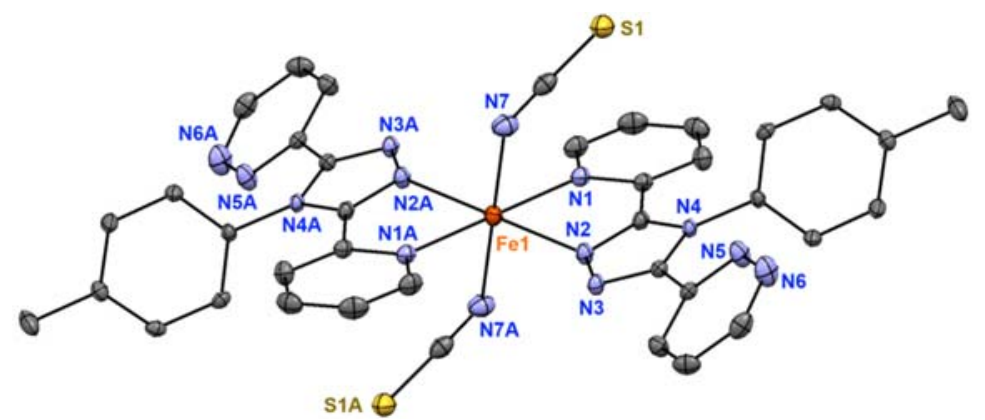

Figure S2. ORTEP representation (50\% probability ellipsoids) of complex [Fe" $\left.\left(\mathrm{L}^{\text {pydzpy }}\right)_{2}(\mathrm{NCSe})_{2}\right](\mathbf{1})$ at $100 \mathrm{~K}$. Hydrogen atoms are omitted for clarity. Symmetry operation $A$ is $-x, 2-y, 1-z$. Note that complex 1 is isomorphous and isostructural to $\left[\mathrm{Fe}^{\prime \prime}\left(\mathrm{L}^{\text {pydzpy }}\right)_{2}(\mathrm{NCSe})_{2}\right](2)$. Bond distances can be found in table S3.
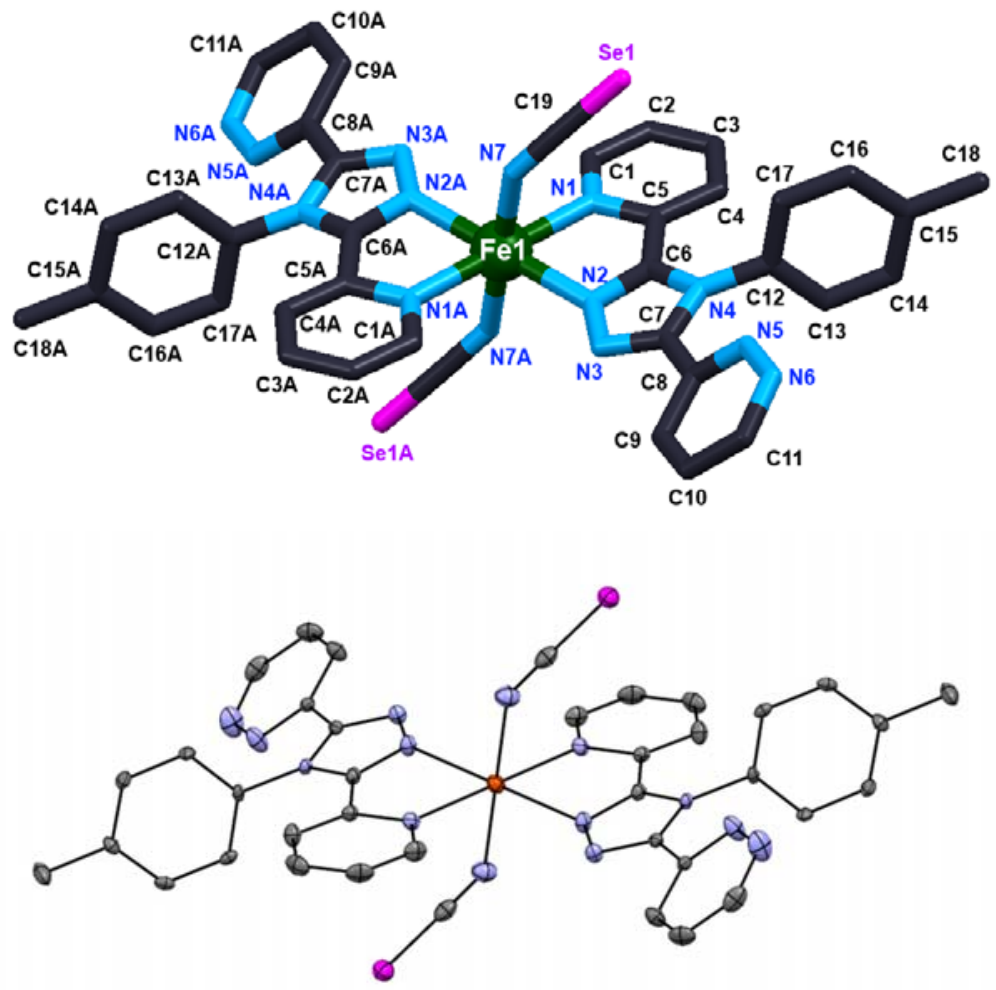

Figure S3. (Top) Perspective view and (bottom) ORTEP representation (50\% probability ellipsoids) of complex $\left[\mathrm{Fe}^{\prime \prime}\left(\mathrm{L}^{\text {pydzpy }}\right)_{2}(\mathrm{NCSe})_{2}\right](2)$ at $100 \mathrm{~K}$. Hydrogen atoms are omitted for clarity. Symmetry operation $\mathrm{A}$ is $-x, 2-y, 1-z$. Note that complex $\left[\mathrm{Fe}^{\prime \prime}\left(\mathrm{L}^{\text {pydzpy }}\right)_{2}(\mathrm{NCS})_{2}\right](2)$ is isomorphous and isostructural to 1 . Bond distances can be found in table S4. 

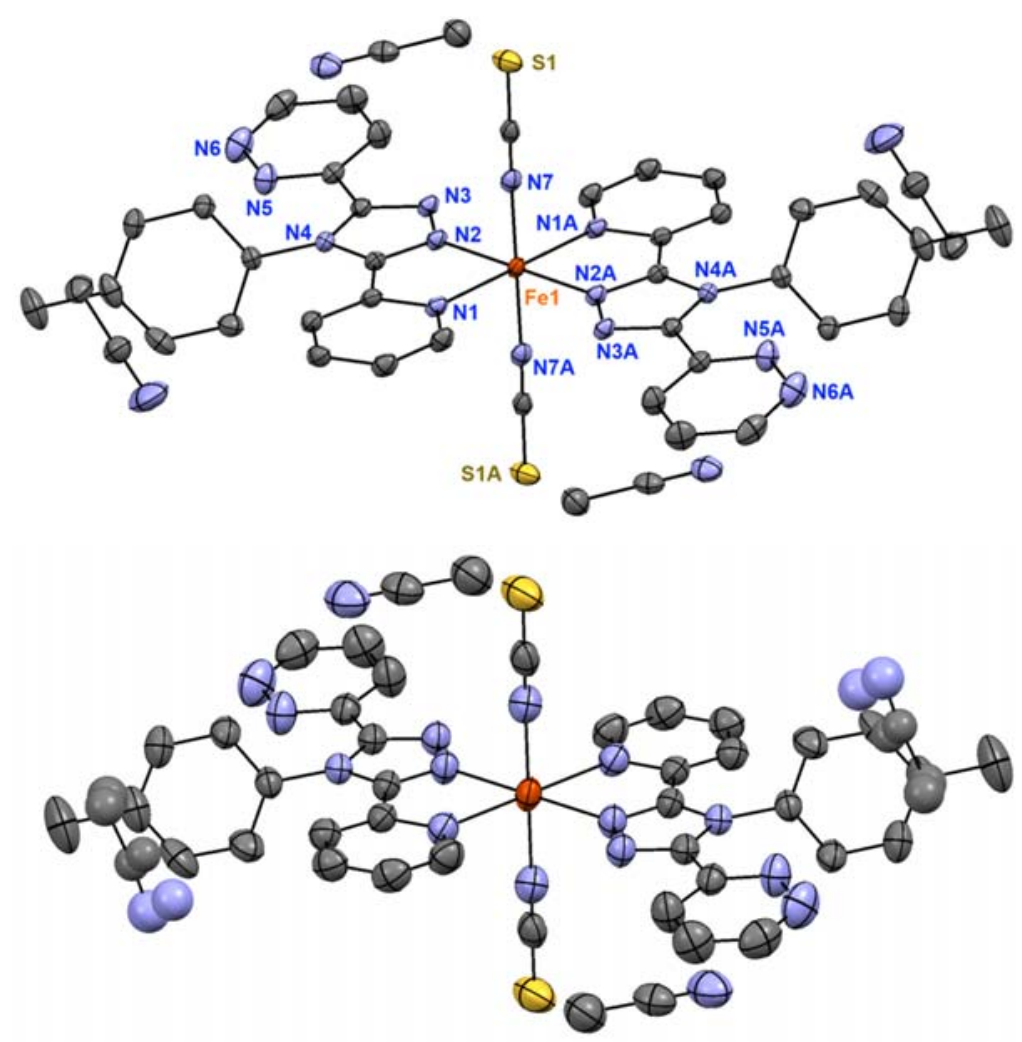

Figure S4. ORTEP representation (50\% probability ellipsoids) of complex $\left[\mathrm{Fe}^{\prime \prime}\left(\mathrm{L}^{\text {pydzpy }}\right)_{2}(\mathrm{NCS})_{2}\right] \cdot 4 \mathrm{CH}_{3} \mathrm{CN}$ $\left(\mathbf{1} \cdot 4 \mathrm{CH}_{3} \mathrm{CN}\right.$ ) at (top) $100 \mathrm{~K}$ and (bottom) $225 \mathrm{~K}$. Hydrogen atoms are omitted for clarity. Symmetry operation $A$ is 1-x, 1-y, 1-z. Note that complex $1 \cdot 4 \mathrm{CH}_{3} \mathrm{CN}$ is isomorphous and isostructural to $\left[\mathrm{Fe}^{\prime \prime}\left(\mathrm{L}^{\text {pydzpy }}\right)_{2}(\mathrm{NCSe})_{2}\right] \cdot 4 \mathrm{CH}_{3} \mathrm{CN}\left(\mathbf{2} \cdot 4 \mathrm{CH}_{3} \mathrm{CN}\right)$. Bond distances can be found in Table $\mathrm{S} 5$ (at $100 \mathrm{~K}$ ) and Table S6 (at $225 \mathrm{~K}$ ). 

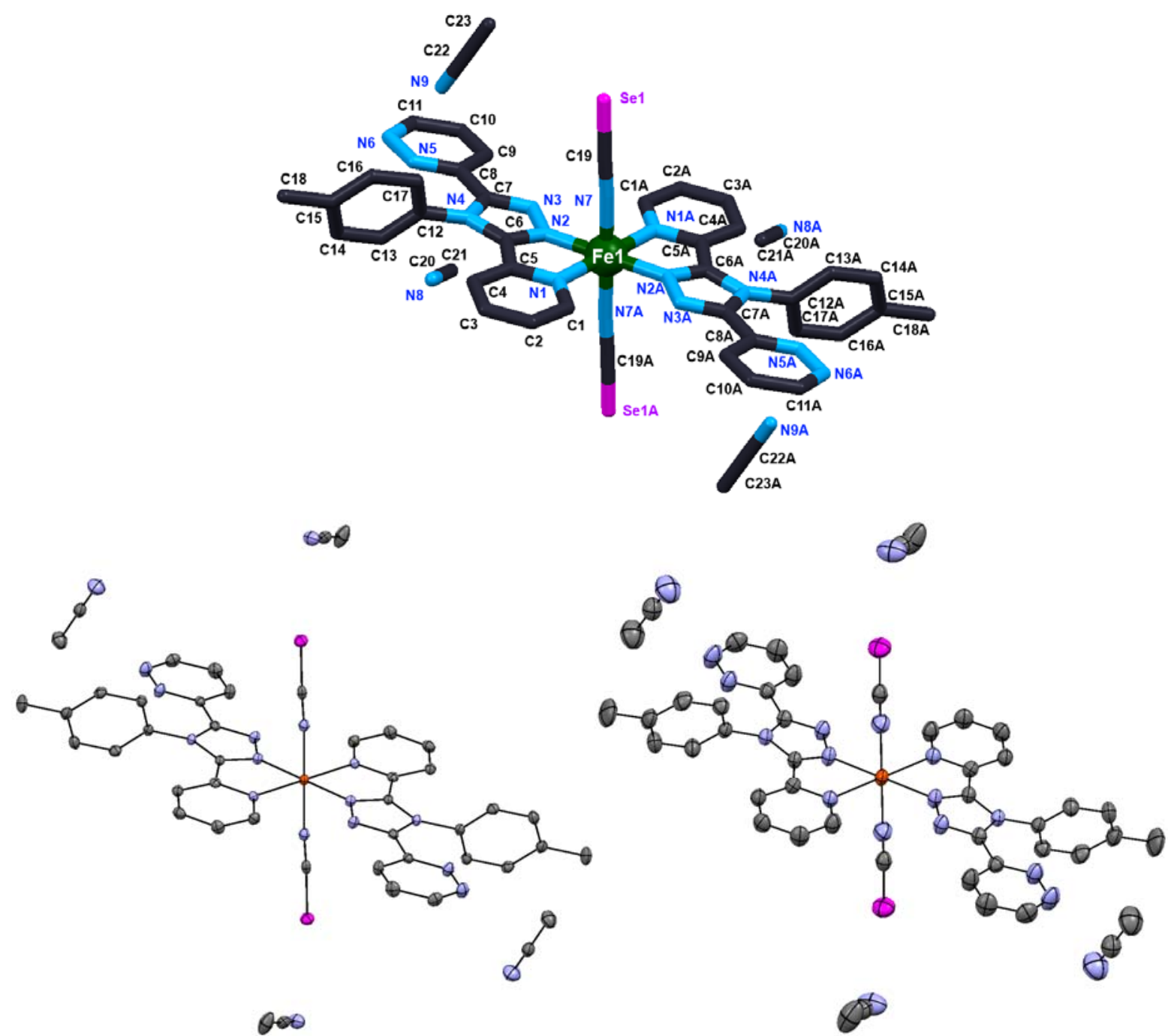

Figure S5. (Top) Perspective view and (bottom) ORTEP representation (50\% probability ellipsoids) of complex [Fe"( ( $\left.\left.{ }^{\text {pydzpy }}\right)_{2}(\mathrm{NCSe})_{2}\right] \cdot 4 \mathrm{CH}_{3} \mathrm{CN}\left(\mathbf{2} \cdot 4 \mathrm{CH}_{3} \mathrm{CN}\right)$ at (bottom left) $100 \mathrm{~K}$ and (bottom right) $270 \mathrm{~K}$. Hydrogen atoms are omitted for clarity. Symmetry operation $A$ is $2-x, 2-y, 1-z$. Note that complex 2. $4 \mathrm{CH}_{3} \mathrm{CN}$ is isomorphous and isostructural to $1 \cdot 4 \mathrm{CH}_{3} \mathrm{CN}$. Bond distances can be found in Table $\mathrm{S} 7$ (at 100 K) and Table S8 (at $270 \mathrm{~K}$ ). 


\section{S1.4. Crystal structure representations of crystal packing}

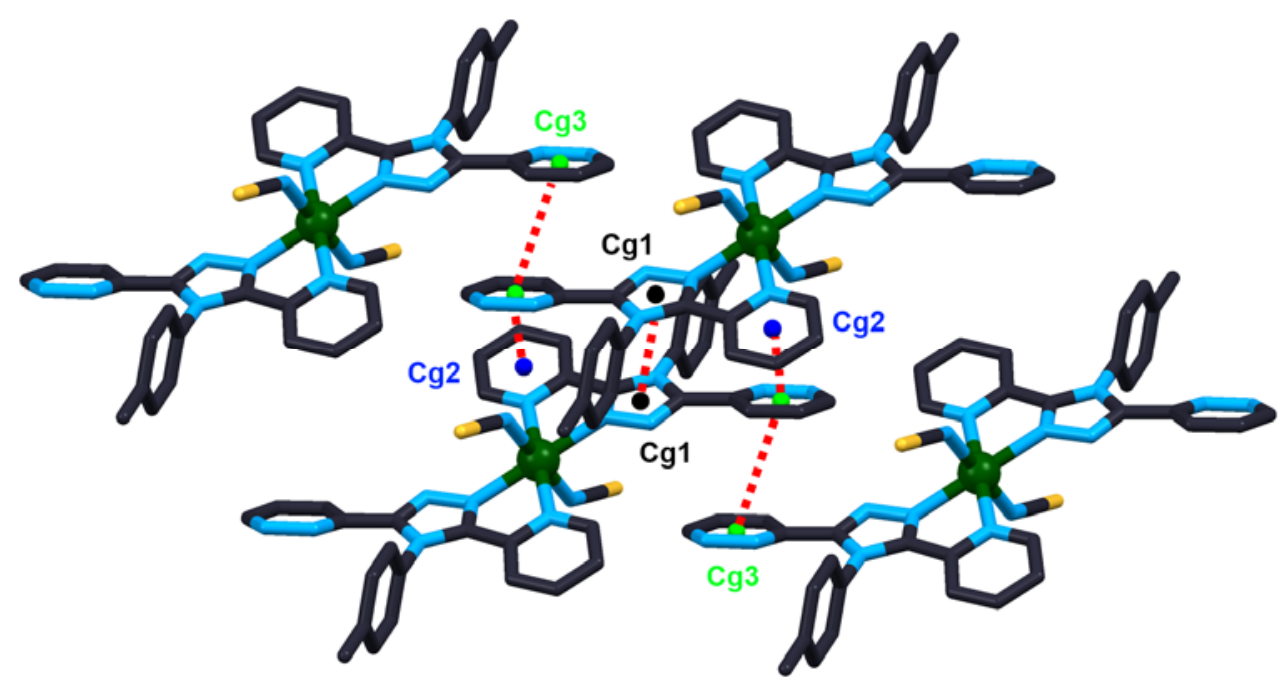

Figure S6. Perspective view of some $\pi \cdots \pi$ intermolecular interactions in [Fe" $\left.\left(\mathrm{L}^{\text {pydzpy }}\right)_{2}(\mathrm{NCS})_{2}\right](\mathbf{1})$ at $100 \mathrm{~K}$. Note that the contacts in complex $\left[\mathrm{Fe}^{\prime \prime}\left(\mathrm{L}^{\text {pydzpy }}\right)_{2}(\mathrm{NCSe})_{2}\right]$ (2) show analogous contacts to 1 . For further information see Table S3 (complex 1) and Table S4 (complex 2). Hydrogen atoms are omitted for clarity. Centroid color code: triazole ring (Cg1, black), pyridine ring (Cg2, blue), pyridazine ring ( $\mathrm{Cg} 3$, green).

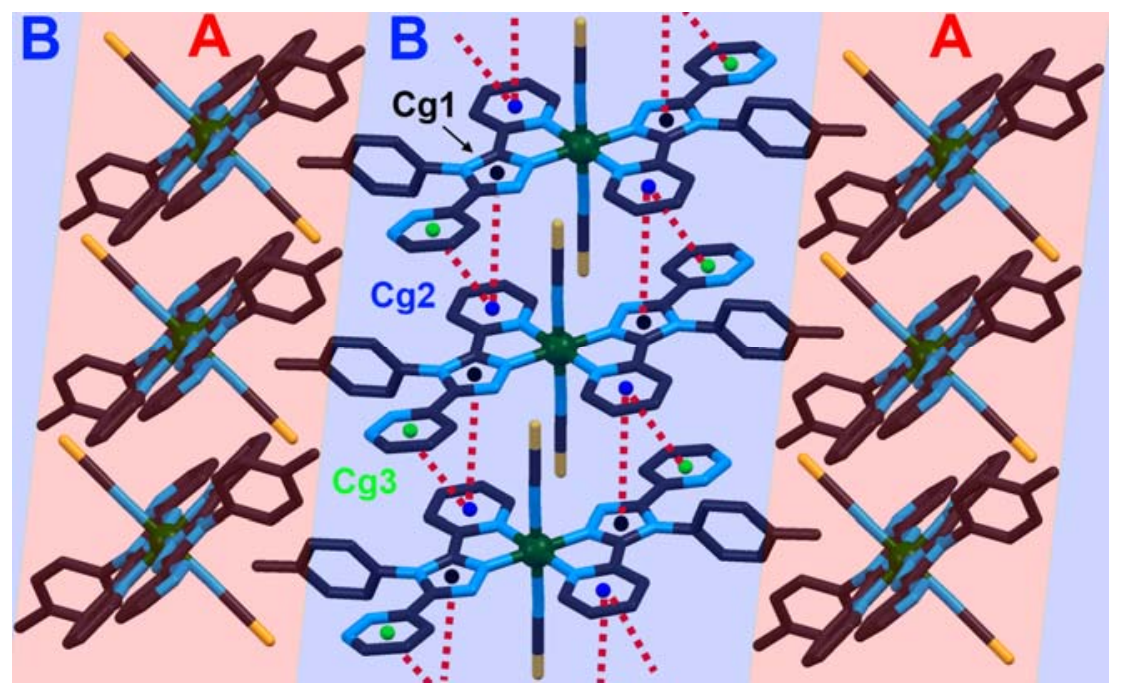

Figure S7. Perspective view of some intermolecular interactions in $\left[\mathrm{Fe}^{\prime \prime}\left(\mathrm{L}^{\text {pydzpy }}\right)_{2}(\mathrm{NCS})_{2}\right] \cdot 4 \mathrm{CH}_{3} \mathrm{CN}\left(\mathbf{1} \cdot 4 \mathrm{CH}_{3} \mathrm{CN}\right)$ at $100 \mathrm{~K}$. The complexes are organized in $A B A B$ layers. This figure corresponds to the same molecules show in Figure $\mathrm{n}$. Molecules within each layer interact through $\pi \cdots \pi$ contacts between coordinated triazole and pyridine rings and uncoordinated pyridazine rings. Molecules between adjacent layers interact via hydrogen bonding contacts between lattice acetonitrile molecules and tolyl rings ( $\mathrm{CH} \cdots \pi$ and $\mathrm{N} \cdots \pi$ ). Note that not all the intermolecular interactions (e.g. those with the A layers) are shown for clarity. For further information see Tables S5 (at $100 \mathrm{~K}$ ) and S6 (at $225 \mathrm{~K}$ ). Hydrogen atoms are omitted for clarity. Centroid color code: triazole ring ( $\mathrm{Cg} 1$, black), pyridine ring ( $\mathrm{Cg} 2$, blue), pyridazine ring ( $\mathrm{Cg} 3$, green). 


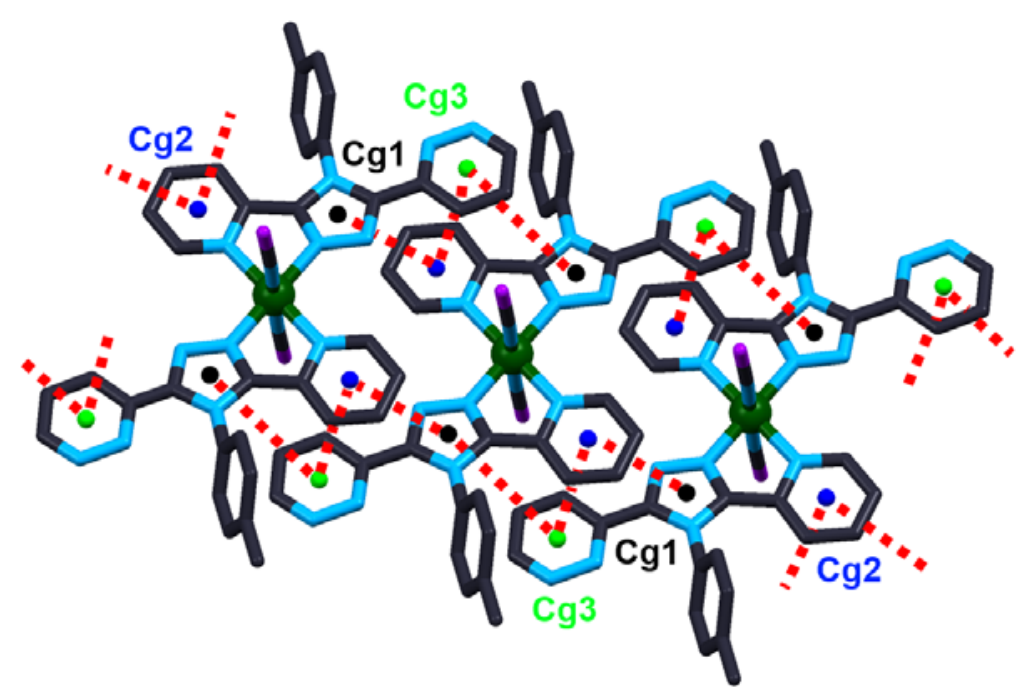

Figure S8. Perspective view of three staggered complexes [ Fe" $\left.\left(\mathrm{L}^{\text {pydzpy }}\right)_{2}(\mathrm{NCSe})_{2}\right] \cdot 4 \mathrm{CH}_{3} \mathrm{CN}\left(2 \cdot 4 \mathrm{CH}_{3} \mathrm{CN}\right)$ within one chain of complexes at $100 \mathrm{~K}$ and the $\pi \cdots \pi$ contacts between these molecules. Hydrogen atoms and lattice acetonitrile molecules are omitted for clarity. For further information see Tables S7 (at $100 \mathrm{~K}$ ) and Table S8 (at $270 \mathrm{~K}$ ). Centroid color code: triazole ring (Cg1, black), pyridine ring (Cg2, blue), pyridazine ring (Cg3, green).

\section{S2. Variable temperature optical microscopy}

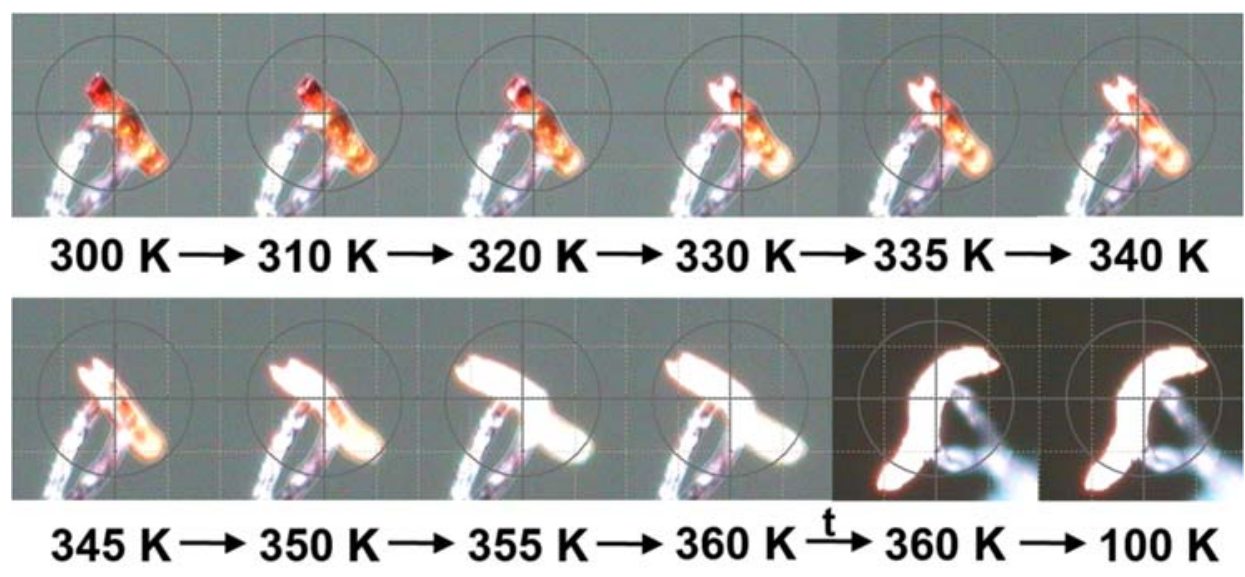

Figure S9. Sequence of photographs of the same single crystal taken at different temperatures for 2. $4 \mathrm{CH}_{3} \mathrm{CN}$. As expected for a SCO compound, the LS state has darker color than the HS state. Based on TGA results on bulk samples (Figure S11, top), $\mathbf{2} \cdot 4 \mathrm{CH}_{3} \mathrm{CN}$ is expected to have desorbed all four lattice acetonitrile molecules per iron(II) center by $353 \mathrm{~K}$. (Note that the last two pictures of $\mathbf{2} \cdot 4 \mathrm{CH}_{3} \mathrm{CN}$ correspond to a $180^{\circ}$ rotation from the previous view). 
S3. Thermogravimetric analysis

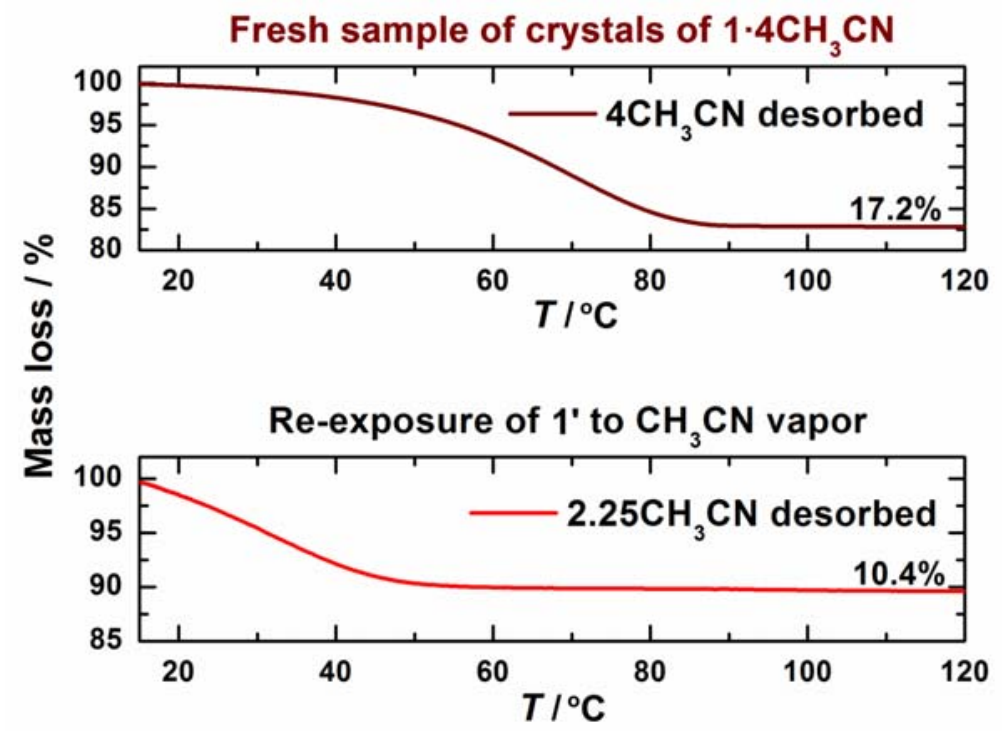

Figure S10. TGA plots of a fresh sample of crystals of $1 \cdot 4 \mathrm{CH}_{3} \mathrm{CN}$ (brown) and $\mathbf{1} \cdot 2.25 \mathrm{CH}_{3} \mathrm{CN}$ (red), obtained after re-exposing the same sample of desolvated $\mathbf{1}^{\prime}$ to $\mathrm{CH}_{3} \mathrm{CN}$ vapor. Calculated mass loss values for: $1 \cdot 4 \mathrm{CH}_{3} \mathrm{CN}(17.0 \%)$ and $1 \cdot 2.25 \mathrm{CH}_{3} \mathrm{CN}(10.3 \%)$. 

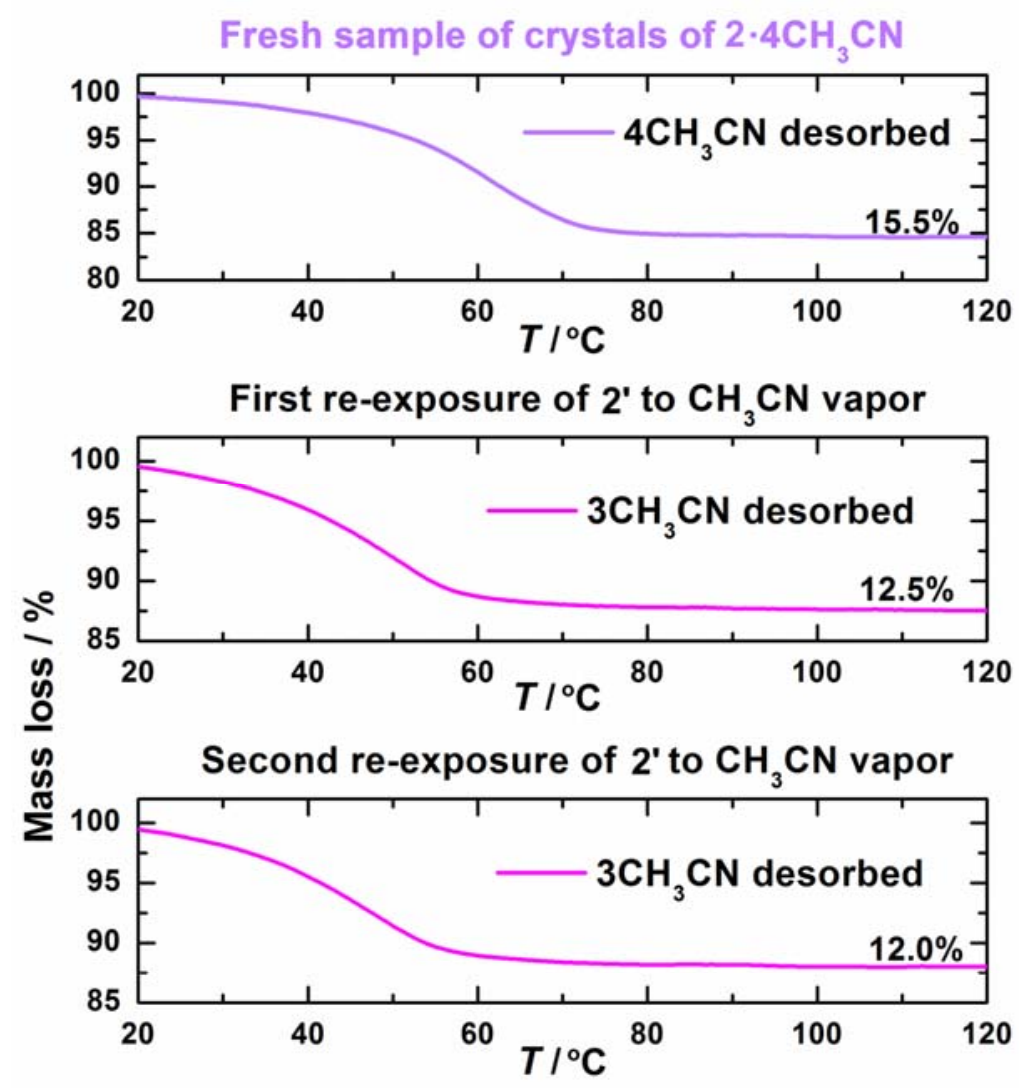

Third re-exposure of 2' to $\mathrm{CH}_{3} \mathrm{CN}$ vapor

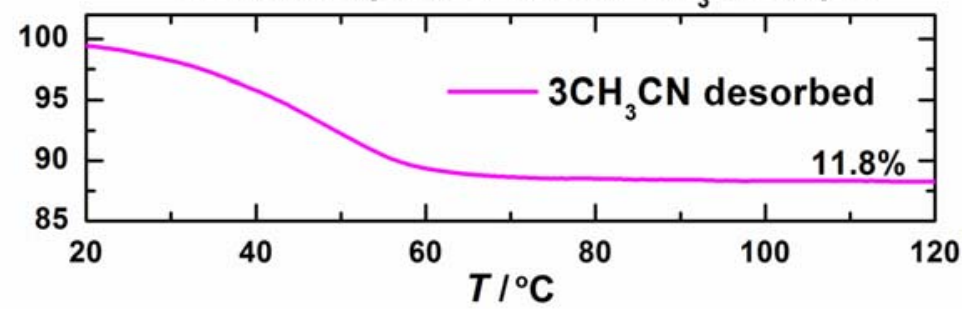

Figure S11. TGA plots of a fresh sample of crystals of $2 \cdot 4 \mathrm{CH}_{3} \mathrm{CN}$ (violet) and $2 \cdot 3 \mathrm{CH}_{3} \mathrm{CN}$ (fuchsia), subsequently obtained by exposing desolvated $\mathbf{2}^{\prime}$ to $\mathrm{CH}_{3} \mathrm{CN}$ vapor up to three times. Calculated mass loss values for: $\mathbf{2} \cdot 4 \mathrm{CH}_{3} \mathrm{CN}(15.5 \%)$ and $\mathbf{2} \cdot 3 \mathrm{CH}_{3} \mathrm{CN}(12.1 \%)$. 
Fresh sample of crystals of $2 \cdot 4 \mathrm{CH}_{3} \mathrm{CN}$
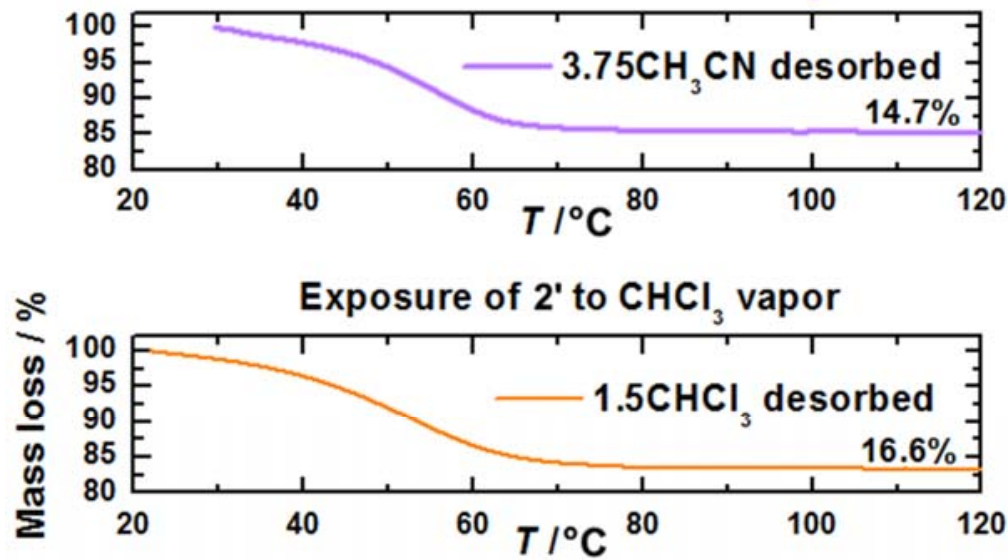

Exposure of 2' to $\mathrm{CH}_{3} \mathrm{CN}$ vapor

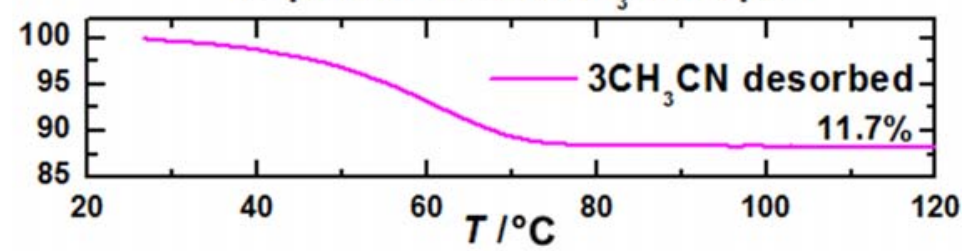

Exposure of $2 \cdot 3 \mathrm{CH}_{3} \mathrm{CN}$ to $\mathrm{CHCl}_{3}$ vapor
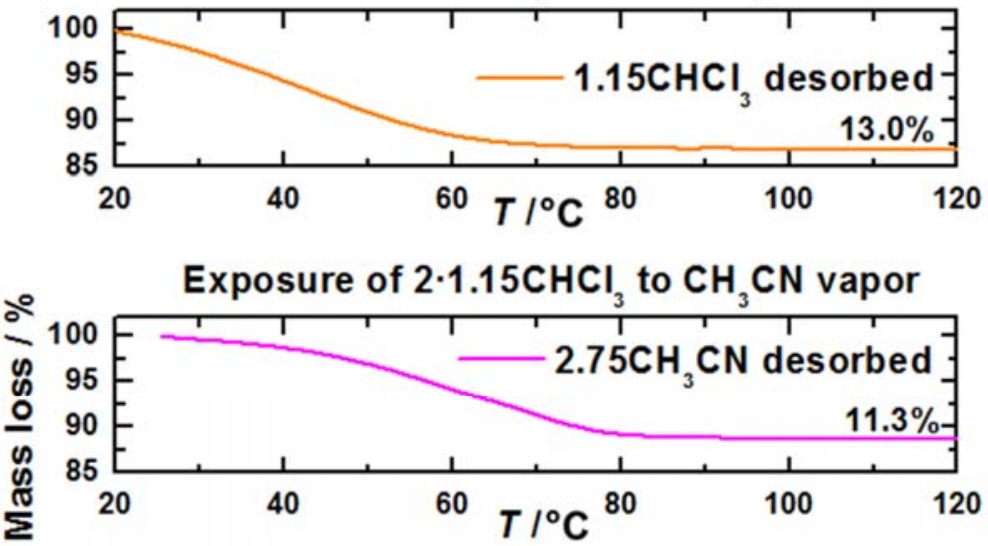

Exposure of $2 \cdot 2.75 \mathrm{CH}_{3} \mathrm{CN}$ to $\mathrm{CHCl}_{3}$ vapor

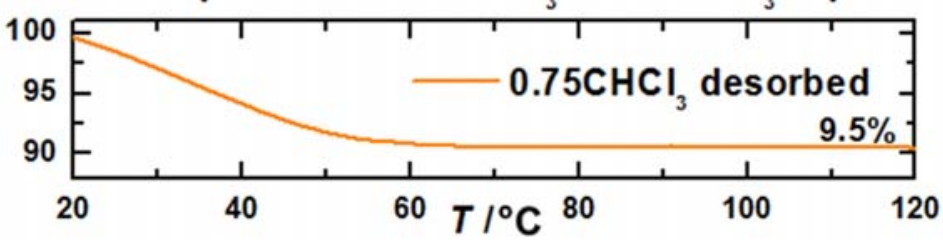

Figure S12. TGA plots of a fresh sample of crystals of $2 \cdot 4 \mathrm{CH}_{3} \mathrm{CN}$ (violet) and the results of exposing the obtained sample to $\mathrm{CH}_{3} \mathrm{CN}$ or $\mathrm{CHCl}_{3}$ vapor. Calculated mass loss values for: $\mathbf{2} \cdot 3.75 \mathrm{CH}_{3} \mathrm{CN}(14.7 \%)$, $\mathbf{2} \cdot 1.5 \mathrm{CHCl}_{3}(16.7 \%), \mathbf{2} \cdot 3 \mathrm{CH}_{3} \mathrm{CN}(12.1 \%), \mathbf{2} \cdot 1.15 \mathrm{CHCl}_{3}(13.3 \%), \mathbf{2} \cdot 2.75 \mathrm{CH}_{3} \mathrm{CN}(11.2 \%)$ and $\mathbf{2} \cdot 0.75 \mathrm{CHCl}_{3}(9.1 \%)$. 

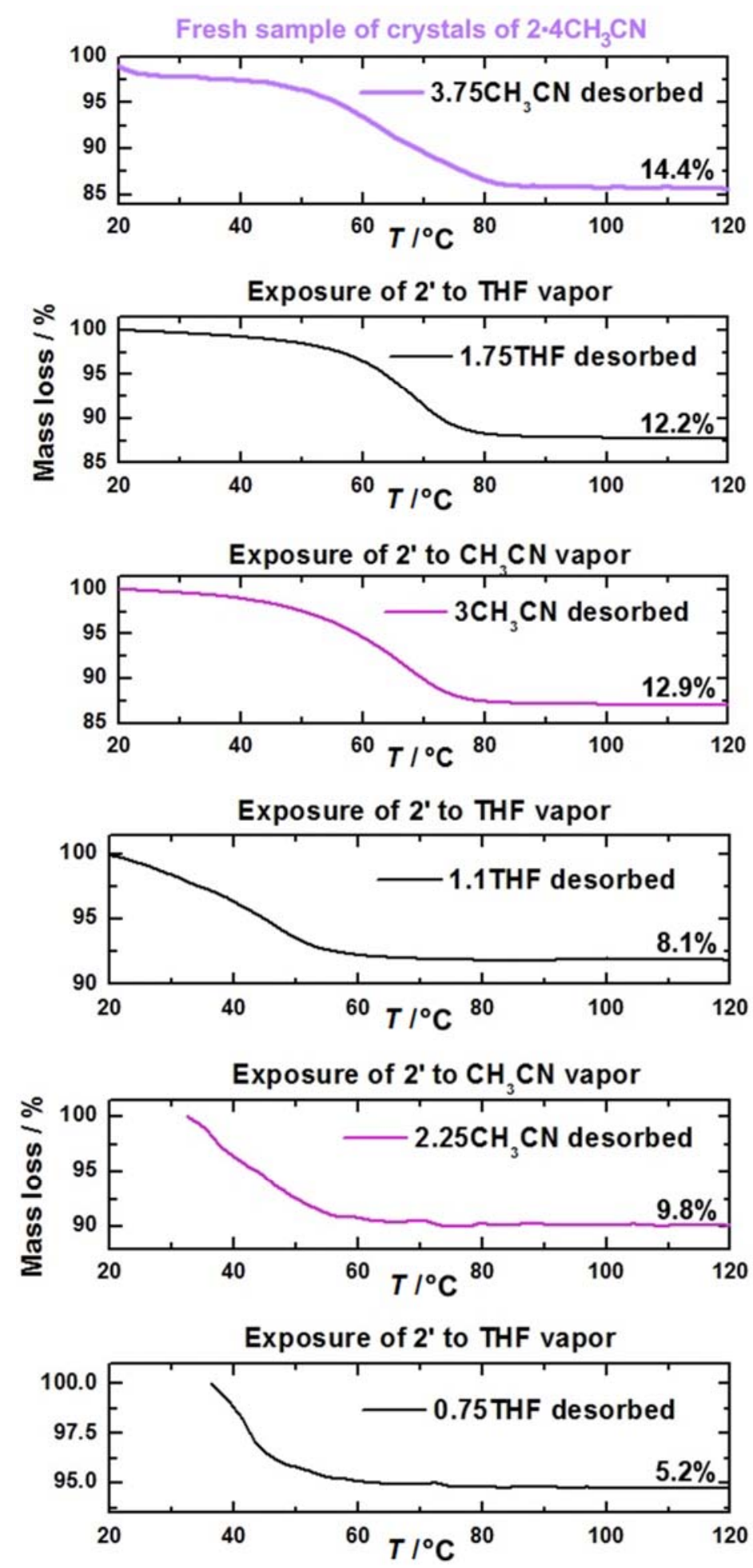

Figure S13. TGA plots of a fresh sample of crystals of $2 \cdot 4 \mathrm{CH}_{3} \mathrm{CN}$ (violet) and the results of exposing the obtained sample to $\mathrm{CH}_{3} \mathrm{CN}$ or THF vapor. Calculated mass loss values for: $\mathbf{2} \cdot 3.75 \mathrm{CH}_{3} \mathrm{CN}(14.7 \%), \mathbf{2} \cdot 1.75 \mathrm{THF}$

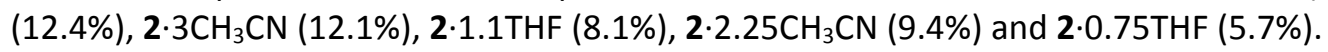



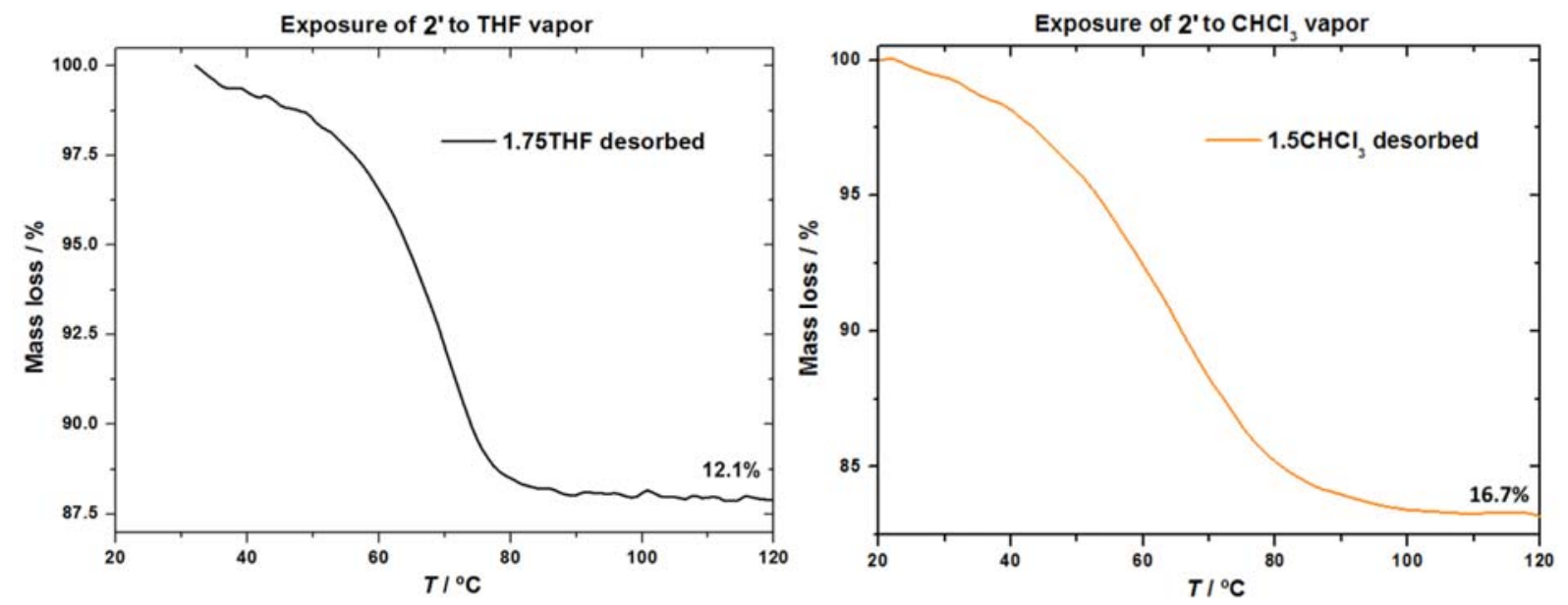

Figure S14. TGA plot of two different samples of 2' exposed to (left, black) THF or (right, orange) $\mathrm{CHCl}_{3}$ vapors of which their magnetic behavior were measured. Calculated mass loss values for 2.1.75THF (12.4\%) and 2.1.5 $\mathrm{CHCl}_{3}$ (16.7\%). 


\section{S4. Powder X-ray diffraction}

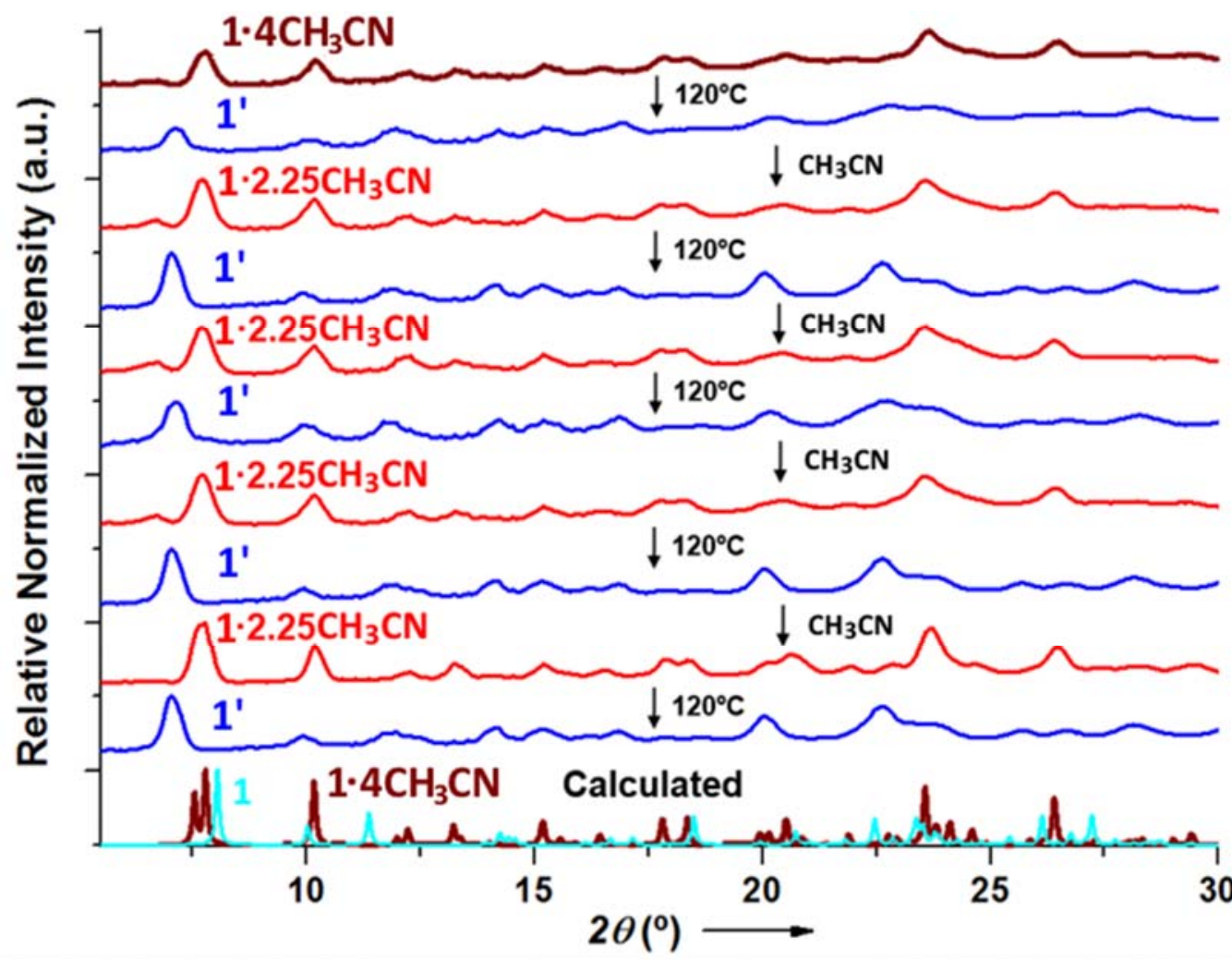

Figure S15. Sequence of PXRD patterns showing the reversible absorption/desorption of $\mathrm{CH}_{3} \mathrm{CN}$ molecules in compound 1' (blue), obtained on the in-house single crystal X-ray diffractometer $(\lambda=1.54184 \AA$ A $)$. All compounds were heated in air, in an oven at $393 \mathrm{~K}$ for 15 minutes; and the desolvated complex 1' was exposed to acetonitrile vapor for 30 minutes. The calculated PXRD spectra of 1 (light blue) and $1 \cdot 4 \mathrm{CH}_{3} \mathrm{CN}$ (brown) are shown at the bottom and were obtained from the SC-XRD data using Mercury with the default setting of FWHM $2 \theta=0.1$ for the width of the peaks. 


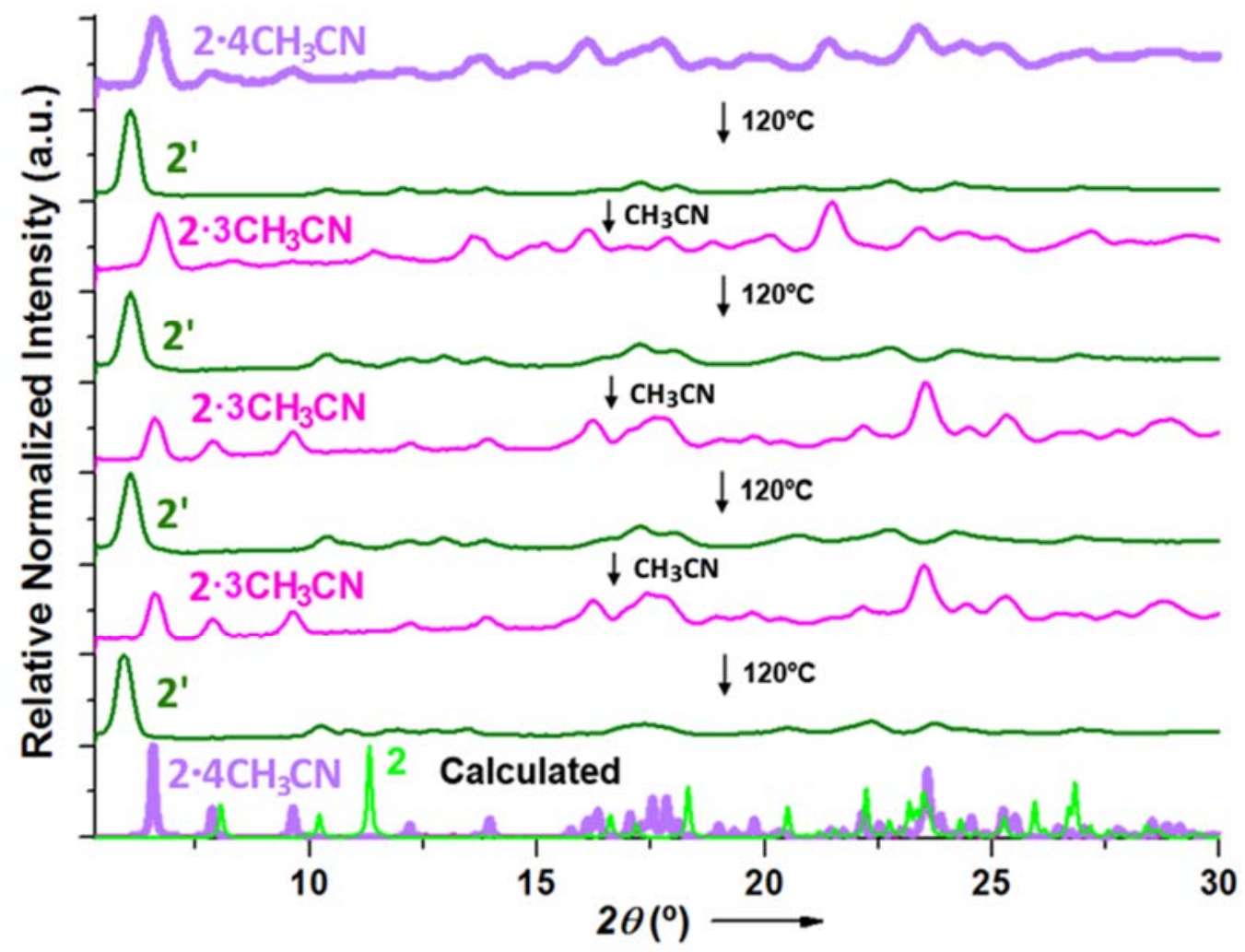

Figure S16. Sequence of PXRD patterns showing the reversible absorption/desorption of acetonitrile molecules in compound 2' (green), obtained on the in-house single crystal X-ray diffractometer ( $\lambda=$ $1.54184 \AA$ A $)$. All compounds were heated in air, in an oven at $393 \mathrm{~K}$ for 15 minutes; and the desolvated complex 2' was exposed to acetonitrile vapor for 30 minutes. The calculated PXRD spectra of $\mathbf{2}$ (light green) and $\mathbf{2} \cdot 4 \mathrm{CH}_{3} \mathrm{CN}$ (violet) are shown at the bottom and were obtained from the SC-XRD data using Mercury with the default setting of FWHM $2 \theta=0.1$ for the width of the peaks. 


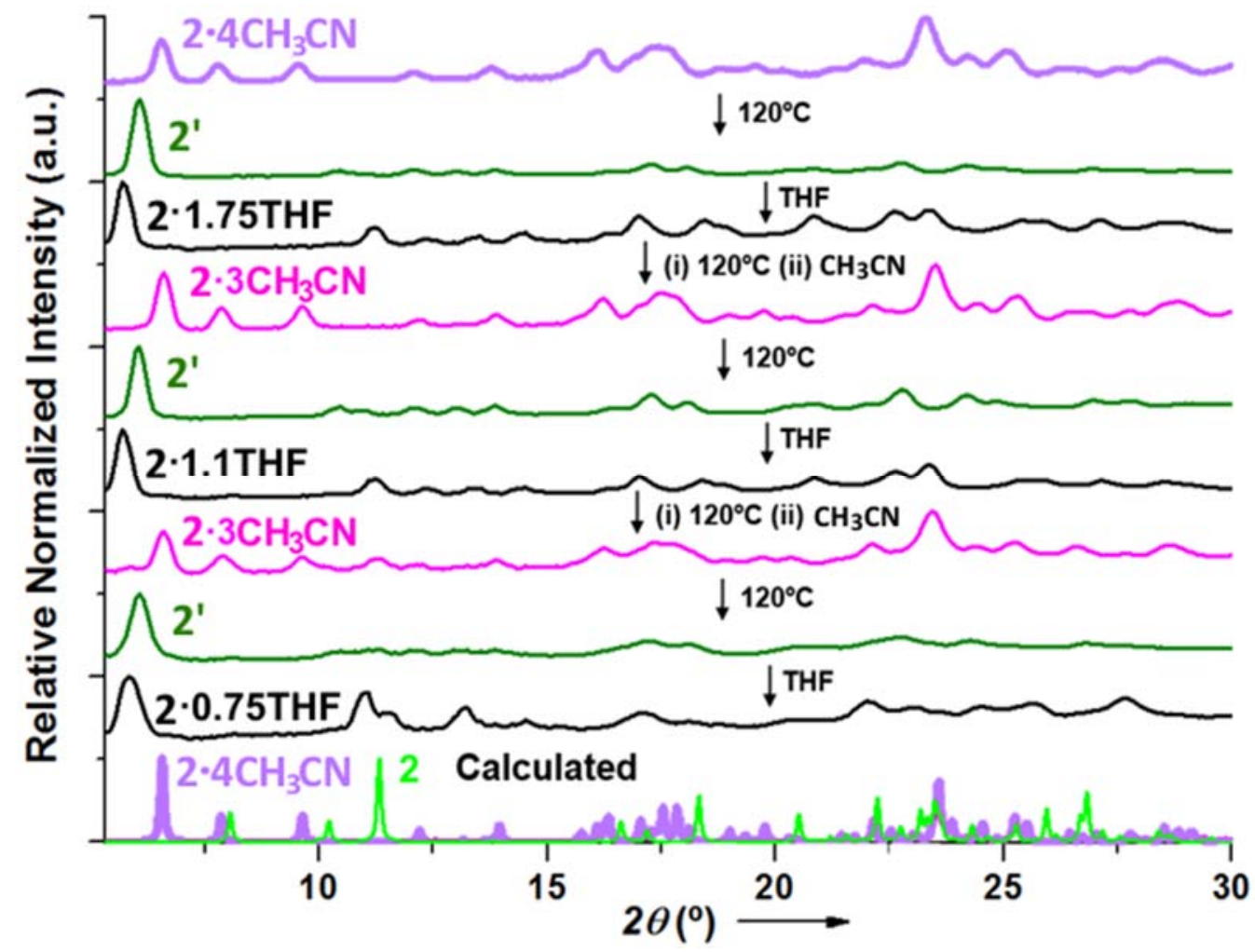

Figure S17. Sequence of PXRD patterns showing the reversible absorption/desorption of acetonitrile and THF molecules in compound 2' (green), obtained on the in-house single crystal X-ray diffractometer ( $\lambda=$ $1.54184 \AA$ A). All compounds were heated in air, in an oven at $393 \mathrm{~K}$ for 15 minutes; and the desolvated complex 2' was exposed to THF vapor for 30 minutes. The calculated PXRD spectra of $\mathbf{2}$ (light green) and 2. $4 \mathrm{CH}_{3} \mathrm{CN}$ (violet) are shown at the bottom and were obtained from the SC-XRD data using Mercury with the default setting of FWHM $2 \theta=0.1$ for the width of the peaks. 


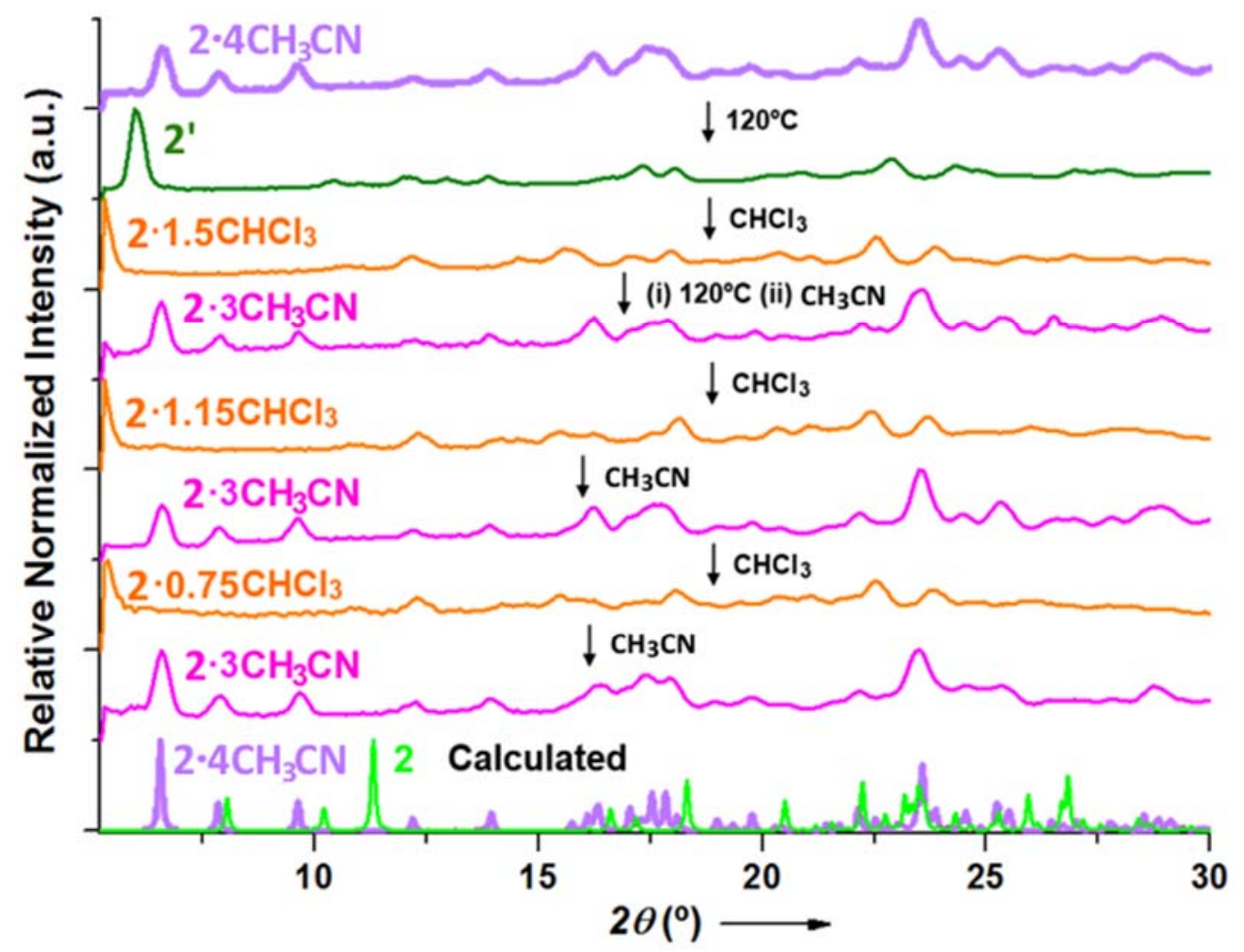

Figure S18. Sequence of PXRD patterns showing the reversible absorption/desorption of acetonitrile and $\mathrm{CHCl}_{3}$ molecules in compound 2' (green), obtained on the in-house single crystal X-ray diffractometer ( $\lambda=$ $1.54184 \AA$ A). All compounds were heated in air, in an oven at $393 \mathrm{~K}$ for 15 minutes; and the desolvated complex 2' was exposed to $\mathrm{CHCl}_{3}$ vapor for 30 minutes. The calculated PXRD spectra of $\mathbf{2}$ (light green) and 2. $4 \mathrm{CH}_{3} \mathrm{CN}$ (violet) are shown at the bottom and were obtained from the SC-XRD data using Mercury with the default setting of FWHM $2 \theta=0.1$ for the width of the peaks. 


\section{S5. Solid-state magnetometry measurements}

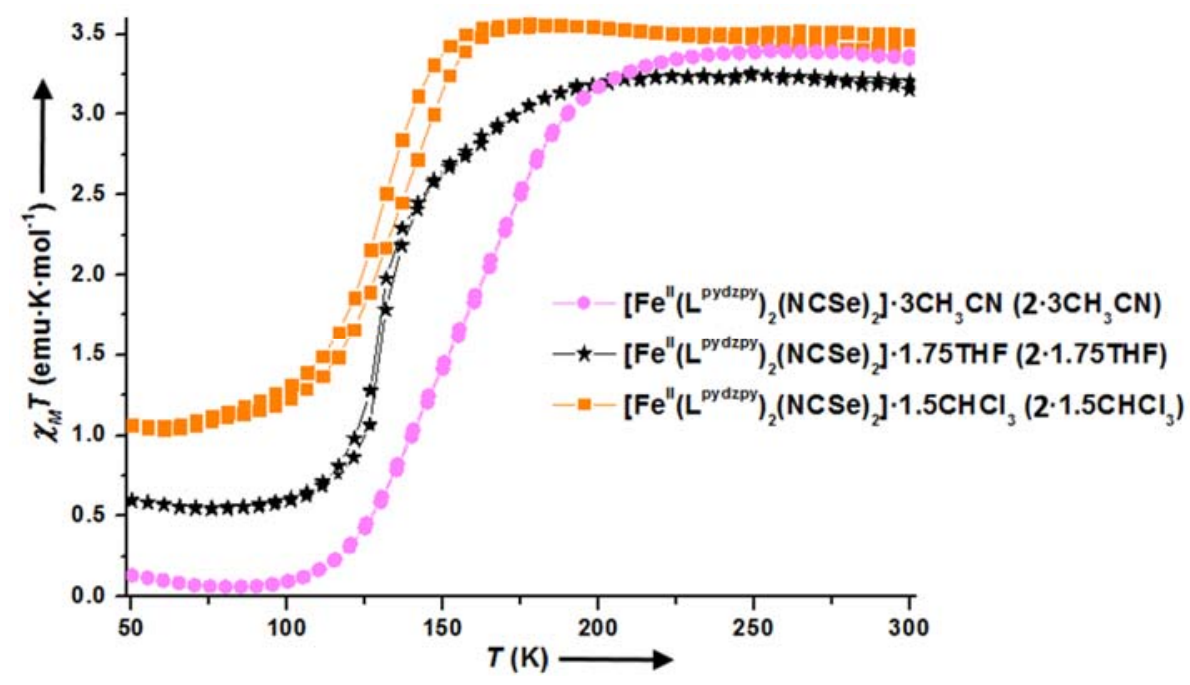

Figures S19. $\chi_{M} T$ versus $T$ plots for $\left[\mathrm{Fe}^{\prime \prime}\left(\mathrm{L}^{\text {pydzpy }}\right)_{2}(\mathrm{NCSe})_{2}\right] \cdot 3 \mathrm{CH}_{3} \mathrm{CN} \quad\left(\mathbf{2} \cdot 3 \mathrm{CH}_{3} \mathrm{CN}\right.$, light pink), $\left[\mathrm{Fe}^{\prime \prime}\left(\mathrm{L}^{\text {pydzpy }}\right)_{2}(\mathrm{NCSe})_{2}\right] \cdot 3 \mathrm{CH}_{3} \mathrm{CN}(\mathbf{2} \cdot 1.75 \mathrm{THF}$, black $)$ and $\left[\mathrm{Fe}^{\prime \prime}\left(\mathrm{L}^{\text {pydzpy }}\right)_{2}(\mathrm{NCSe})_{2}\right] \cdot 3 \mathrm{CH}_{3} \mathrm{CN}\left(\mathbf{2} \cdot 1.5 \mathrm{CHCl}_{3}\right.$, orange $)$. The temperature ramp rate between data points, with data collected in settle mode at $5 \mathrm{~K}$ intervals, was $5 \mathrm{~K} \cdot \mathrm{min}^{-1}$ (see Experimental Section). 